ESAIM: M2AN 47 (2013) 1287-1314

DOI: $10.1051 / \mathrm{m} 2 \mathrm{an} / 2013068$
ESAIM: Mathematical Modelling and Numerical Analysis

www.esaim-m2an.org

\title{
NUMERICAL ANALYSIS OF PARALLEL REPLICA DYNAMICS
}

\author{
Gideon Simpson ${ }^{1}$ AND Mitchell Luskin ${ }^{1}$
}

\begin{abstract}
Parallel replica dynamics is a method for accelerating the computation of processes characterized by a sequence of infrequent events. In this work, the processes are governed by the overdamped Langevin equation. Such processes spend much of their time about the minima of the underlying potential, occasionally transitioning into different basins of attraction. The essential idea of parallel replica dynamics is that the exit distribution from a given well for a single process can be approximated by the distribution of the first exit of $N$ independent identical processes, each run for only $1 / N$-th the amount of time. While promising, this leads to a series of numerical analysis questions about the accuracy of the exit distributions. Building upon the recent work in [C. Le Bris, T. Lelièvre, M. Luskin and D. Perez, Monte Carlo Methods Appl. 18 (2012) 119-146], we prove a unified error estimate on the exit distributions of the algorithm against an unaccelerated process. Furthermore, we study a dephasing mechanism, and prove that it will successfully complete.
\end{abstract}

Mathematics Subject Classification. 60H35, 65C20, 65C30, 70K70, 74S60.

Received April 3, 2012. Revised January 31, 2013.

Published online July 9, 2013.

\section{INTRODUCTION}

Parallel replica dynamics (ParRep) is a numerical tool first introduced by Voter in [26] (see also [22, 27]) for accelerating the simulation of stochastic processes characterized by a sequence of infrequent, but rapid, transitions from one state to another. A standard and important problem in which such a separation of scales is present is the migration of defects through a crystalline lattice; see [22] and references therein for examples.

Roughly, the idea behind parallel replica dynamics is as follows. Suppose a trajectory spends time $t$ in a particular state, before transitioning into another. Furthermore, assume $t$ is large, relative to the scale of the time step discretization. We wish to avoid directly simulating a single realization for time $t$. We approximate the simulation of a single trajectory for time $t$ with $N$ independent copies, each simulated for time $t / N$, and follow the particular trajectory that escapes first. This holds out the promise for a linear speedup with the number of independent realizations we are able to simulate.

Of course, this is not exact, and error is introduced. A particular concern is error in the exit distributions of the system as it migrates from one state to another - does ParRep disrupt the state to state dynamics? Inspired by the tools proposed in [4], we prove an error estimate on the exit distributions over a single "cycle" of ParRep (the transition from one state to the next).

Keywords and phrases. Accelerated dynamics, rare events, parallel replica.

1 School of Mathematics, University of Minnesota, 206 Church St SE, 127 Vincent Hall, Minneapolis MN 55455, USA.

gsimpson@umn.edu; luskin@umn.edu 


\subsection{The algorithm}

We assume the system we wish to accelerate evolves according to the overdamped Langevin equation,

$$
\mathrm{d} X_{t}=-\nabla V\left(X_{t}\right) \mathrm{d} t+\sqrt{2 \beta^{-1}} \mathrm{~d} B_{t}, \quad X_{t} \in \mathbb{R}^{n},
$$

where $B_{t}$ is a Wiener process and $\beta$ is proportional to inverse temperature. Though ParRep was originally developed for the Langevin equations, it is readily adapted to this problem.

We next assume that our system is such that $V$ has a denumerable set of local minima, $x_{j}, j=1,2, \ldots$ For each minima, we associate a set $W_{j} \subset \mathbb{R}^{n}$, the "well." $W_{j}$ could be the basin of attraction of $x_{j}$; if $y(t)$ solves the ODE

$$
\dot{y}=-\nabla V(y), \quad y(0)=y_{0} \in \mathbb{R}^{n}
$$

then

$$
W_{j}=\left\{y_{0}: \lim _{t \rightarrow+\infty} y(t)=x_{j}\right\} .
$$

However, this definition is not essential; for the sake of our analysis, $W_{j}$ need only be a bounded set in $\mathbb{R}^{n}$ with sufficiently regular boundary.

This motivates defining the well selection function,

$$
\mathcal{S}: \mathbb{R}^{n} \rightarrow \mathbb{N}
$$

which identifies the basin associated with a given position. Associated with this is the "coarse grained" trajectory,

$$
\mathcal{S}_{t} \equiv \mathcal{S}\left(X_{t}\right)
$$

which only identifies the present well.

If the wells are "deep" with well-defined minima, then $X_{t}$ will infrequently transition from one to another. Such a well corresponds to a metastable state. Much of the simulation time will be spent waiting for a jump to occur. The goal of ParRep is to reduce this computational expense by providing a satisfactory approximation of the form

$$
\mathcal{S}_{t} \approx \mathcal{S}_{t}^{\text {ParRep }} .
$$

In other words, we are willing to sacrifice information about where the trajectory is within each well, for the sake of rapidly computing the sequence of wells the trajectory visits.

We now describe the ParRep algorithm in the following steps: the decorrelation step; the dephasing step; and the parallel step. These steps are diagrammed in Figures 1 and 2. We assume that the reference process $X_{t}^{\text {ref }}$ enters well $W_{j}$ at time $t_{\text {sim }}$.

A. Decorrelation Step: Let $X_{t}^{\text {ref }}$ evolve under (1.1) for $t_{\mathrm{sim}} \leq t \leq t_{\mathrm{sim}}+t_{\mathrm{corr}}$.

- If

$$
\mathcal{S}\left(X_{t}^{\mathrm{ref}}\right)=\mathcal{S}\left(X_{t_{\mathrm{sim}}}^{\mathrm{ref}}\right)
$$

for all $t_{\mathrm{sim}} \leq t \leq t_{\mathrm{sim}}+t_{\text {corr }}$, then time advances to $t_{\mathrm{sim}}+t_{\text {corr }}$ and proceed.

- Otherwise, denote the first exit time from the well,

$$
T=\inf \left\{t \mid \mathcal{S}\left(X_{t_{\mathrm{sim}}+t}^{\mathrm{ref}}\right) \neq \mathcal{S}\left(X_{t_{\mathrm{sim}}}^{\mathrm{ref}}\right)\right\}
$$

and time advances to $t_{\mathrm{sim}}+T$. Return to the beginning of the decorrelation step in the new well.

B. Dephasing Step: In conjunction with the decorrelation step, we launch $N$ replicas with starting positions drawn from distribution $\mu_{\text {phase }}^{0}$. These are run for $t_{\text {phase }}$ amount of time, the dephasing time. If at any time before $t_{\text {phase }}$ a replica leaves the well, it is restarted. A replica has successfully dephased if it remains in the well for all of $t_{\text {phase. }}$ 


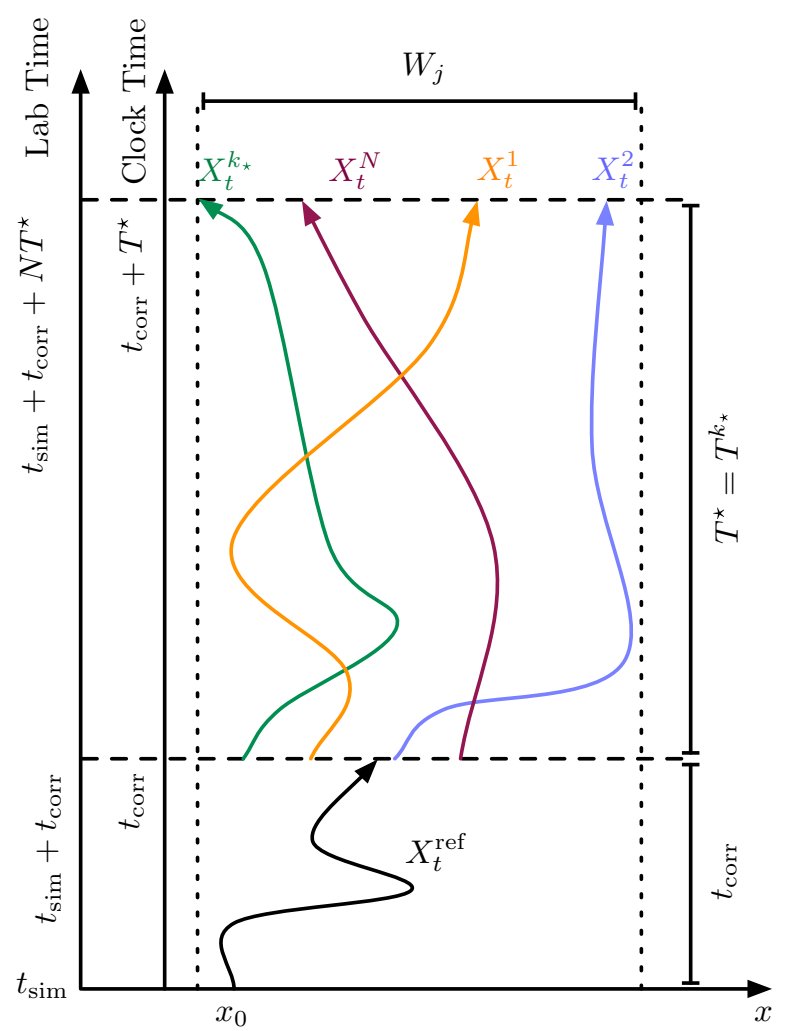

Figure 1. An illustration of the decorrelation and parallel steps of the ParRep algorithm in the case that the reference walker never leaves well $W_{j} . X_{t}^{k_{\star}}$ is the first process to exit the well, doing so at the computer time $t_{\text {corr }}+T^{\star}$. This is then translated into the lab, or physical, time $t_{\text {sim }}+t_{\text {corr }}+N T^{\star}$. See Figure 2 for an illustration of a dephasing step.

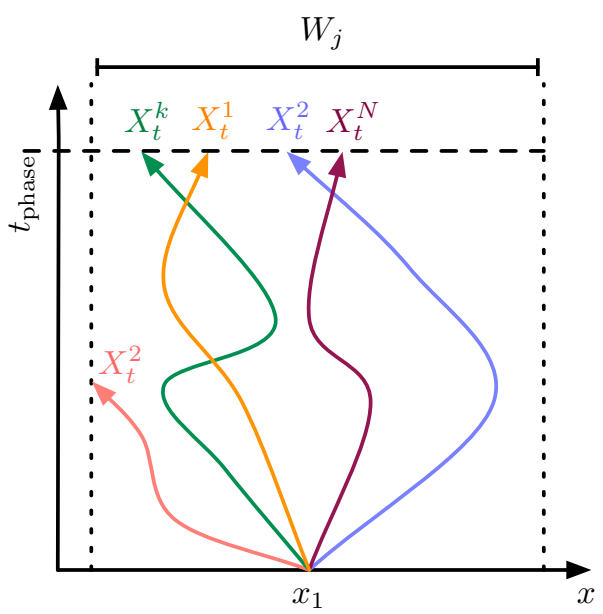

FiguRE 2. An illustration of a dephasing step for the ParRep algorithm. In this implementation, the replicas all start from the same position; $\mu_{\text {phase }}^{0}=\delta_{x_{1}}$. When $X_{t}^{2}$ leaves before $t_{\text {phase }}$, it is relaunched from the same position. 
At the completion of the decorrelation and dephasing steps, assuming the reference walker has not exited, we have $N$ independent walkers with the same distribution. We discard the reference process. If at any time during the dephasing process the reference walker leaves the well, the dephasing process terminates and the replicas are discarded.

C. Parallel Step: We now let the $N$ replicas evolve independently and define

$$
\begin{aligned}
k_{\star} & =\underset{k}{\operatorname{argmin}} T^{k}, \\
X_{t}^{\star} & =X_{t}^{k_{\star}}, \\
T^{\star} & =T^{k_{\star}} .
\end{aligned}
$$

The system advances to the next well:

$$
\begin{gathered}
t_{\mathrm{sim}} \mapsto t_{\mathrm{sim}}+t_{\mathrm{corr}}+N T^{\star} \\
X_{t_{\mathrm{sim}}}^{\mathrm{ref}}+t_{\mathrm{corr}}+N T^{\star}=X_{T^{\star}}^{\star} .
\end{gathered}
$$

Finally, we return to the decorrelation step.

This is a different dephasing algorithm than described in [4]. There, after the decorrelation step, the replicas are initiated at the the position of the reference process and run for $t_{\text {phase }}$. The simulation clock is not advanced, and replicas are replaced as need be should they exit the well. Our implementation has the advantage that no processor sits idle.

The reader may wonder why we would want to have a distinguished reference process - why not relaunch the reference process, as we would a replica, should it exit? We retain this feature to allow for realizations where the process is in a well for a very short period, far less than the decorrelation time. These correlated events, such as recrossings, appear in serial simulations and should be preserved. One may also ask why we discard the reference process. This is to simplify the analysis, as it permits us to declare that the $N$ replicas are drawn from the same distribution when the parallel step begins.

In addition to the choice of $t_{\text {corr }}$ and $t_{\text {phase }}$, there is also the question of what $\mu_{\text {phase }}^{0}$ should be. Again, there is significant flexibility. One possibility is to allow the reference process to evolve for some amount of time, and then the replicas could be launched from its position. A method used in practice is to find a local minima associated with the well, and initiate the replicas from that position, [21]. We emphasize that the dephasing mechanism need not depend on any information associated with the reference process.

In principle, ParRep offers a nearly linear speedup with the number of independent replicas, provided $t_{\text {corr }}$ is short relative to the typical exit time. With the explosion in the availability of distributed computing clusters, parallel replica dynamics is an attractive tool for studying infrequent event processes.

\subsection{Main results}

The essential aspects of a process undergoing infrequent transitions are

- How often does it transition from one state to another?

- What state does it transition to?

These properties are captured in $\mathcal{S}_{t}$. To assess how well $\mathcal{S}_{t}^{\text {ParRep }}$ approximates it, we are motivated to first consider the exit distribution of a process, and how well it is preserved. In [4], the authors proposed a rigorous framework in which to study ParRep. The purpose of this study is to unify those ideas and assess the total error, over a single cycle of ParRep, as a function of the parameters.

Note. For brevity, we shall now take $t_{\mathrm{sim}}=0$ and $W_{j}=W$. Throughout our paper, we shall assume:

- $W \subset \mathbb{R}^{n}$ is bounded;

- $\partial W$ is sufficiently smooth; 
- $V$ is sufficiently smooth on $\bar{W}$.

Though $W$ need not correspond to a basin of attraction, we shall continue to call it a well.

To motivate our results, we introduce some important objects. Let $\mu_{t}$ denote the law of $X_{t}$, conditioned on having not left the well:

$$
\mu_{t}(A)=\mathbb{P}^{\mu_{0}}\left[X_{t} \in A \mid T>t\right]=\frac{\mathbb{P}^{\mu_{0}}\left[X_{t} \in A, T>t\right]}{\mathbb{P}^{\mu_{0}}[T>t]}
$$

The above expression is the probability of finding the processes, $X_{t}$, in the set $A \subset W$, at time $t$, conditioned on the exit time from the well, $T$, being beyond $t$, and $X_{0}$ being initially distributed by $\mu_{0}$. Additional details on our notation are given below, in Section 1.4. Under certain assumptions, the limit

$$
\lim _{t \rightarrow \infty} \mu_{t}=\nu,
$$

exists. $\nu$ is the quasistationary distribution (QSD) and characterizes the long term survivors of (1.1) in well $W$. The properties of $\nu$ are reviewed for the reader in Section 2.

In the following theorems, we shall refer to "admissible distributions." This class is quite broad and includes the Dirac distribution. It is defined and explored in subsequent sections. First, we have the following result on the convergence of the exit distribution of $X_{t}$.

Theorem 1.1 (Convergence to the QSD). Assume $\mu_{0}$ is admissible. There exist positive constants $\lambda_{2}>\lambda_{1}, C$ and $\underline{t}$, such that for all $t \geq \underline{t}$ and bounded and measurable $f(\tau, \xi): \mathbb{R}^{+} \times \partial W \rightarrow \mathbb{R}$ we have

$$
\left|\mathbb{E}^{\mu_{t}}\left[f\left(T, X_{T}\right)\right]-\mathbb{E}^{\nu}\left[f\left(T, X_{T}\right)\right]\right| \leq C\|f\|_{L^{\infty}} \mathrm{e}^{-\left(\lambda_{2}-\lambda_{1}\right) t} .
$$

The constant $C$ is independent of $t$ and $f$.

Taking $t$ sufficiently large so as to make this small corresponds to the satisfactory completion of the decorrelation step; this reflects (1.8). We give a more precise statement of this theorem at the beginning of Section 3, after introducing some additional notation in Section 2. This result also plays a role in studying the dephasing step. The constants $C$ and $\underline{t}$ depend on $\mu_{0}, V$, and the geometry of the well. We will use the notation $C_{\text {phase }}$ and $C_{\text {corr }}$, and $\underline{t}_{\text {phase }}$ and $\underline{t}_{\text {corr }}$ to distinguish the constants induced by the dephasing and decorrelation steps.

The next result ensures that the dephasing step terminates successfully:

Theorem 1.2 (Dephasing process). For an admissible distribution $\mu_{\mathrm{phase}}^{0}$ and $t_{\text {phase }} \geq \underline{t}_{\mathrm{phase}}$ :

A. Dephasing produces $N$ independent replicas with distributions $\mu_{\text {phase; }}$;

B. Given any $\epsilon>0$, by taking $t_{\text {phase }} \geq \underline{t}_{\text {phase }}$,

$$
\left|\mathbb{E}^{\mu_{\text {phase }}}\left[f\left(T^{k}, X_{T^{k}}^{k}\right)\right]-\mathbb{E}^{\nu}\left[f\left(T, X_{T}\right)\right]\right| \leq C_{\text {phase }} \mathrm{e}^{-\left(\lambda_{2}-\lambda_{1}\right) t_{\text {phase }}}\|f\|_{L^{\infty}}
$$

C. The expected number of times a replica is relaunched is finite.

Next, the error in the parallel step cascading from the dephasing step can be controlled:

Theorem 1.3 (Parallel error). Given $t_{\text {phase }} \geq \underline{t}_{\text {phase }}$, let

$$
\epsilon_{\text {phase }} \equiv C_{\text {phase }} \mathrm{e}^{-\left(\lambda_{2}-\lambda_{1}\right) t_{\text {phase }}},
$$

and assume the dephasing step has produced $N$ i.i.d. replicas drawn from distribution $\mu_{\text {phase }}$.

Then the exit time converges to an exponential law, with parameter $N \lambda_{1}$,

$$
\left|\mathbb{P}^{\mu_{\text {phase }}}\left[T^{\star}>t\right]-\mathrm{e}^{-N \lambda_{1} t}\right| \leq N \epsilon_{\text {phase }}\left(1+\epsilon_{\text {phase }}\right)^{N-1} \mathrm{e}^{-N \lambda_{1} t} .
$$


If we additionally assume that $N \epsilon_{\text {phase }}\left(1+\epsilon_{\text {phase }}\right)^{N-1}<1$, then the hitting point distribution is asymptotically independent of the exit time

$$
\left|\mathbb{P}^{\mu_{\text {phase }}}\left[X_{T^{\star}}^{\star} \in A \mid T^{\star}>t\right]-\int_{A} \mathrm{~d} \rho\right| \lesssim \frac{N^{2} \epsilon_{\text {phase }}\left(1+\epsilon_{\text {phase }}\right)^{N-1}}{1-N \epsilon_{\text {phase }}\left(1+\epsilon_{\text {phase }}\right)^{N-1}},
$$

where $\rho$ is the hitting point density and $A \subset \partial W$.

Thus, for $t_{\text {phase }}$ large enough, we achieve the ideal factor of $N$ speedup and we do not disrupt the hitting point distribution too much. The reader may find the $N$ dependence in the error terms to be disconcerting, but it can easily be controlled by taking $t_{\text {phase }} \gtrsim \log N /\left(\lambda_{2}-\lambda_{1}\right)$. We will return to this in the discussion. We also note that there is a slight abuse of notation in the above expressions. The superscripts, $\nu$ and $\mu_{\text {phase }}$, should be interpreted as $N$-tensor products, with a distinct realization drawn for each replica.

A more detailed statement of this theorem, with explicit constants, is given at the beginning of Section 5 . The hitting point density $\rho$ is defined by $(2.14)$.

However, Theorem 1.3 is only a comparison between the parallel step and the QSD. Our final result is a comparison between the ParRep algorithm, including decorrelation, dephasing and parallel steps, with an unaccelerated, serial process:

Theorem 1.4 (ParRep error). Let $X_{t}^{\mathrm{s}}$ denote the unaccelerated (serial) process and $X_{t}^{\mathrm{p}}$ denote the ParRep process, and let both the serial process and the reference process be initially distributed under $\mu_{0}$, an admissible distribution. Furthermore, assume the replicas are initialized from $\mu_{\text {phase }}^{0}$, also an admissible distribution.

Given $t_{\text {corr }} \geq \underline{t}_{\text {corr }}$ and $t_{\text {phase }} \geq \underline{t}_{\text {phase }}$, let

$$
\begin{aligned}
\epsilon_{\text {corr }} & =C_{\text {corr }} \mathrm{e}^{-\left(\lambda_{2}-\lambda_{1}\right) t_{\text {corr }}}, \\
\epsilon_{\text {phase }} & =C_{\text {phase }} \mathrm{e}^{-\left(\lambda_{2}-\lambda_{1}\right) t_{\text {phase }}} .
\end{aligned}
$$

Letting $T^{\mathrm{s}}$ and $T^{\mathrm{p}}$ denote the physical exit times, we have

$$
\begin{aligned}
& \left|\mathbb{P}^{\mu_{0}}\left[T^{\mathrm{s}}>t\right]-\mathbb{P}^{\mu_{0}}\left[T^{\mathrm{p}}>t\right]\right| \\
& \quad \lesssim\left[\epsilon_{\text {corr }}+N \epsilon_{\text {phase }}\left(1+\epsilon_{\text {phase }}\right)^{N-1}\right] \mathrm{e}^{-\lambda_{1}\left(t-t_{\text {corr }}\right)_{+} .}
\end{aligned}
$$

If, in addition, $t_{\text {corr }}$ is sufficiently large such that $\epsilon_{\mathrm{corr}}<1$, then for $A \subset \partial W$,

$$
\begin{aligned}
& \left|\mathbb{P}^{\mu_{0}}\left[X_{T^{\mathrm{s}}}^{\mathrm{s}} \in A \mid T^{\mathrm{s}}>t\right]-\mathbb{P}^{\mu_{0}}\left[X_{T^{\mathrm{p}}}^{\mathrm{p}} \in A \mid T^{\mathrm{p}}>t\right]\right| \\
& \quad \lesssim \frac{\epsilon_{\text {corr }}+N^{2} \epsilon_{\text {phase }}\left(1+\epsilon_{\text {phase }}\right)^{N-1}}{1-\epsilon_{\text {corr }}}
\end{aligned}
$$

Thus, over a single cycle, the error in ParRep can be approximately decomposed as

$$
\text { Error }=\text { Decorrelation error }+ \text { Parallel error(Dephasing error), }
$$

where we view the parellel error as a function of the dephasing error. The speedup can be seen when $T^{\mathrm{p}}$ is given further consideration. When $T^{\mathrm{p}}>t_{\text {corr }}, T^{\mathrm{p}}=N T^{\star}+t_{\text {corr }}$ where $T^{\star}$ is the exit time of the particular replica which escapes first. There will be no speedup if the exit is before $t_{\text {corr }}$.

\subsection{Outline of the Paper}

In Section 2, we review some important results for (1.1). Our main Theorems are proven in Sections 3, 4, and 5 . We then discuss our results in Section 6. Some additional calculations appear in the appendix. 


\subsection{Notation}

Random variables, such as the position, $X_{t}$, and the exit time from the well, $T$, will appear in capital letters. Deterministic values, such as $x, t, t_{\text {corr }}$, etc. will be lower case. We will frequently use indicator functions in our analysis, which we write as $1_{A}$, with $A$ indicating the set on which the value is one.

We are often interested in probabilities and expectations of solutions of $X_{t}$ solving (1.1), and its exit time $T$ from some region $W$. When we write

$$
\mathbb{E}^{x}\left[f\left(T, X_{T}\right)\right] \text { or } \mathbb{P}^{x}[T \geq t]=\mathbb{E}^{x}\left[1_{T \geq t}\right]
$$

the superscript $x$ indicates that $x$ is the initial condition of $X_{t} ; X_{0}=x$, and the expectation and probability are then taken with respect to the underlying Wiener measure of $B_{t}$.

When $X_{0}$ is given by some distribution $\mu_{0}$ over $W$, we write

$$
\mathbb{E}^{\mu_{0}}\left[f\left(T, X_{T}\right)\right] \equiv \int_{W} \mathbb{E}^{x}\left[f\left(T, X_{T}\right)\right] \mathrm{d} \mu_{0}(x) .
$$

When we write a conditional expectation with respect to distribution $\mu_{0}$, we mean

$$
\mathbb{E}^{\mu_{0}}\left[f\left(T, X_{T}\right) \mid T>t\right] \equiv \frac{\mathbb{E}^{\mu_{0}}\left[f\left(T, X_{T}\right) 1_{T>t}\right]}{\mathbb{P}^{\mu_{0}}[T>t]}
$$

For the reader more accustomed to the computational physics literature,

$$
\mathbb{E}^{\mu_{0}}\left[\mathcal{O}\left(X_{t}\right)\right]=\langle\mathcal{O}(t)\rangle .
$$

It is helpful to explicitly include the starting distribution, $\mu_{0}$ associated with the process $X_{t}$, to avoid any ambiguity.

When we write $f \lesssim g$, we mean that there exists a constant $C>0$ such that $f \leq C g$, but that the constant is not noteworthy.

\section{Preliminary Results}

Before proceeding to our main results on ParRep, we review some important results on the overdamped Langevin equation. These results are where our regularity assumptions on $V, W$, and $\partial W$ are needed.

Two essential tools in our study of (1.1) are the Feynman-Kac formula and the quasistationary distribution, which we briefly review here; see [4] for additional details. First, let us recall the Feynman-Kac formula which relates solutions of a parabolic equation with corresponding elliptic operator

$$
L \equiv-\nabla V \cdot \nabla+\beta^{-1} \Delta
$$

to solutions of (1.1).

Proposition 2.1 (Prop. 1 of [4]). On the parabolic domain $W \times \mathbb{R}^{+}$, let $v$ solve

$$
\begin{aligned}
\partial_{t} v & =L v, \\
\left.v\right|_{\partial W} & =\phi: \partial W \rightarrow \mathbb{R}, \\
v(t=0) & =v_{0}: W \rightarrow \mathbb{R} .
\end{aligned}
$$

Then,

$$
v(t, x)=\mathbb{E}^{x}\left[1_{T \leq t} \phi\left(X_{T}\right)\right]+\mathbb{E}^{x}\left[1_{T>t} v_{0}\left(X_{t}\right)\right] .
$$


To say a bit more about the elliptic operator $L$, recall the invariant measure of (1.1):

$$
\mathrm{d} \mu \equiv Z^{-1} \exp (-\beta V(x)) \mathrm{d} x,
$$

where $Z$ is the appropriate normalization. We introduce the Hilbert space $L_{\mu}^{2}$, with inner product

$$
\langle f, g\rangle_{\mu} \equiv \int f g \mathrm{~d} \mu \text {. }
$$

An elementary calculation shows that $L$ is self adjoint and negative definite with respect to this inner product when supplemented with homogeneous Dirichlet boundary conditions on $\partial W$. Standard functional analysis and elliptic theory tell us that $L$ has infinitely many eigenvalue/eigenfunction pairs $\left(\lambda_{k}, u_{k}\right)$; the eigenvalues can be ordered

$$
0>-\lambda_{1}>-\lambda_{2} \geq-\lambda_{3} \geq \ldots ;
$$

and the eigenfunctions form a complete orthonormal basis for $L_{\mu}^{2}(W)$. In addition, the ground state, $u_{1}$, is unique and positive. For details, see, for example, $[13,14,16]$. The $\lambda_{1}$ and $\lambda_{2}$ appearing in our theorems are precisely the first two eigenvalues.

When solving (2.2) with $\phi=0$, the solution can be expressed as

$$
v(x, t)=\sum_{k=1}^{\infty} \mathrm{e}^{-\lambda_{k} t}\left\langle v_{0}, u_{k}\right\rangle_{\mu} u_{k} .
$$

Out of this spectral problem, we build the norm

$$
\|f\|_{H_{\mu}^{s}}^{2} \equiv \sum_{k=1}^{\infty} \lambda_{k}^{s}\left|\left\langle f, u_{k}\right\rangle_{\mu}\right|^{2} .
$$

This generalizes to measures

$$
\left\|\mu_{0}\right\|_{H_{\mu}^{s}}^{2} \equiv \sum_{k=1}^{\infty} \lambda_{k}^{s}\left|\int u_{k} \mathrm{~d} \mu_{0}\right|^{2}
$$

and to sequences, $\mathbf{a}=\left(a_{1}, a_{2}, \ldots\right)$

$$
\|\mathbf{a}\|_{H_{\mu}^{s}}^{2} \equiv \sum_{k=1}^{\infty} \lambda_{k}^{s}\left|a_{k}\right|^{2} .
$$

If $\mu_{0}$ has an Radon-Nikodym derivative with respect to $\mu,(2.7)$ and (2.8) agree. We then define the function spaces,

$$
H_{\mu}^{s}=\left\{v \in \mathscr{S}(W)^{\prime} \mid\|v\|_{H_{\mu}^{s}}<\infty\right\}
$$

where $\mathscr{S}$ is the set of smooth functions with support in $W$, and $\mathscr{S}^{\prime}$ is its dual. We also define the projection operator, $P_{\mathcal{I}}$, where $\mathcal{I} \subset \mathbb{N}$,

$$
P_{\mathcal{I}} f=\sum_{k \in \mathcal{I}}\left\langle f, u_{k}\right\rangle_{\mu} u_{k}
$$

Having introduced these spaces and norms, we can now clarify what was meant by the term admissible distribution used in the introduction. In this work, a distribution will be admissible with respect to $W$ if $\operatorname{supp} \mu_{0} \subset W$, and for some $s \geq 0,\left\|\mu_{0}\right\|_{H_{\mu}^{-s}}<\infty$.

The aforementioned quasistationary distribution of (1.1) associated with the set $W$ is closely related to the spectral structure of $L$. The QSD, $\nu$, is a time independent probability measure satisfying, for all measurable $A \subset W$ and $t>0$ :

$$
\nu(A)=\frac{\int_{W} \mathbb{P}^{x}\left[X_{t} \in A, t<T\right] \mathrm{d} \nu}{\int_{W} \mathbb{P}^{x}[t<T] \mathrm{d} \nu}=\mathbb{P}^{\nu}\left[X_{t} \in A \mid t<T\right] .
$$

The QSD measure $\nu$ exists and 
Proposition 2.2 (Prop. 2 of [4]).

$$
\mathrm{d} \nu=\frac{u_{1} \mathrm{~d} \mu}{\int_{W} u_{1} \mathrm{~d} \mu}=\frac{u_{1} \mathrm{e}^{-\beta V} \mathrm{~d} x}{\int_{W} u_{1} \mathrm{e}^{-\beta V} \mathrm{~d} x} .
$$

We refer the reader to, amongst others, $[5-7,19,20,25]$ for additional details on the QSD. The utility of the QSD stems from the property that if $X_{0}$ is distributed according to $\nu$, then:

Proposition 2.3 (Prop. 3 of [4]). Let $\phi: \partial W \rightarrow \mathbb{R}$ be smooth. Then for $t>0$

$$
\mathbb{E}^{\nu}\left[1_{T<t} \phi\left(X_{T}\right)\right]=\mathbb{P}^{\nu}[T<t] E^{\nu}\left[\phi\left(X_{T}\right)\right]=\left(1-\mathrm{e}^{-\lambda_{1} t}\right) \int_{\partial_{W}} \phi \mathrm{d} \rho
$$

where the exit density is given by

$$
\mathrm{d} \rho=-\frac{1}{\lambda_{1} \beta} \nabla \frac{\mathrm{d} \nu}{\mathrm{d} x} \cdot \mathbf{n} d S_{x}=-\frac{\nabla\left(u_{1} \mathrm{e}^{-\beta V}\right) \cdot \mathbf{n}}{\lambda_{1} \beta \int_{W} u_{1} \mathrm{e}^{-\beta V} \mathrm{~d} x} d S_{x},
$$

with $\mathbf{n}$ the outward pointing normal and $d S_{x}$ the surface measure.

In words, $T$ is exponentially distributed with parameter $\lambda_{1}$, and the first hitting point is independent of the first hitting time. Being initially distributed according to $\nu$ is, in a sense, ideal. As shown by Proposition 5 of [4], were this the case for the replicas, the parallel step of ParRep would be exact. In practice, $X_{0}$ is never distributed by $\nu$, and it is the propagation of this error that we explore.

Many of these quantities can be reformulated in terms of the Fokker-Planck equation for density $p^{x}(t, y)$, $x \in W$,

$$
\begin{gathered}
\partial_{t} p^{x}=L^{*} p^{x}=\nabla_{y} \cdot\left(p^{x} \nabla V+\beta^{-1} \nabla p^{x}\right) \\
\left.p^{x}\right|_{\partial W}=0, \quad p_{0}^{x}=\delta_{x}(y) .
\end{gathered}
$$

Though we will not make use of this, the reader more accustomed to Fokker-Planck may find it helpful to re-express various quantities in terms of $p^{x}$. With regard to exit distributions,

$$
\begin{aligned}
\mathbb{E}^{x}\left[\phi\left(X_{T}\right) 1_{T<t}\right] & =\int_{0}^{t} \int_{\partial W}-\phi(y) \beta^{-1} \nabla p^{x} \cdot \mathbf{n} \mathrm{d} S_{y} \\
\mathbb{P}^{x}[t<T] & =\int_{t}^{\infty} \int_{\partial W}-\beta^{-1} \nabla p^{x} \cdot \mathbf{n} \mathrm{d} S_{y}=\int_{W} p^{x}(t, y) \mathrm{d} y .
\end{aligned}
$$

These can be integrated against the density of the QSD, $\frac{\mathrm{d} \nu}{\mathrm{d} y}$, which solves $L^{*} \frac{\mathrm{d} \nu}{\mathrm{d} x}=-\lambda_{1} \frac{\mathrm{d} \nu}{\mathrm{d} y}$, to obtain

$$
p^{\nu}(y, t)=\mathrm{e}^{-\lambda_{1} t} \frac{\mathrm{d} \nu}{\mathrm{d} y}
$$

as a particular solution of the Fokker-Planck equation. This directly shows the independence of exit time and hitting point. Substituting into the above integrals reproduces Proposition 2.3. 


\section{Convergence to the QSD - Proof of Theorem 1.1}

In this section we prove Theorem 1.1, which we first restate with more detail:

Theorem 3.1 (Convergence to the QSD). Given $s \geq 0$, let $\mu_{0}$ be a distribution with supp $\mu_{0} \subset W$ and $\left\|\mu_{0}\right\|_{H_{\mu}^{-s}}<\infty$. There exists

$$
\underline{t} \gtrsim\left\{\left\|P_{[2, \infty)} \mu_{0}\right\|_{H_{\mu}^{-s}} / \int u_{1} \mathrm{~d} \mu_{0}\right\}^{4 /(n+2 s)}
$$

such that for all $t \geq \underline{t}$ and for all bounded and measurable $f(\tau, \xi): \mathbb{R}^{+} \times \partial W \rightarrow \mathbb{R}$

$$
\begin{aligned}
& \left|\mathbb{E}^{\mu_{t}}\left[f\left(T, X_{T}\right)\right]-\mathbb{E}^{\nu}\left[f\left(T, X_{T}\right)\right]\right| \\
& \lesssim\|f\|_{L^{\infty}}\left(\int u_{1} \mathrm{~d} \mu_{0}\right)^{-1} \underline{t}^{-n / 4-s / 2} \mathrm{e}^{-\left(\lambda_{2}-\lambda_{1}\right)(t-\underline{t})}\left\|P_{[2, \infty)} \mu_{0}\right\|_{H_{\mu}^{-s}} .
\end{aligned}
$$

This is a refinement of Proposition 6 from [4], which now admits initial distributions which lack an $L^{2}$ RadonNikodym derivative. Indeed, for appropriate $s, \mu_{0}$ can be a Dirac distribution. Though this is a parabolic flow which will instantaneously regularize such rough data, it is essential to an analysis of ParRep as one often wants to use Dirac mass initial conditions.

In addition to this result, we present an extension which is essential to obtaining the results in Section 5 on the parallel step.

\subsection{Proof of Theorem 3.1}

Proof. We first write

$$
\mathbb{E}^{\mu_{t}}\left[f\left(T, X_{T}\right)\right]=\int_{W} \mathbb{E}^{x}\left[f\left(T, X_{T}\right)\right] \mathrm{d} \mu_{t}=\int_{W} F(x) \mathrm{d} \mu_{t}
$$

where we have defined $F(x) \equiv \mathbb{E}^{x}\left[f\left(T, X_{T}\right)\right]$. Thus,

$$
\mathbb{E}^{\mu_{t}}\left[f\left(T, X_{T}\right)\right]=\frac{\int_{W} \mathbb{E}^{x}\left[F\left(X_{t}\right) 1_{T>t}\right] \mathrm{d} \mu_{0}}{\int_{W} \mathbb{E}^{x}\left[1_{T>t}\right] \mathrm{d} \mu_{0}} .
$$

Applying Feynman-Kac, (2.3), to this,

$$
\mathbb{E}^{\mu_{t}}\left[f\left(T, X_{T}\right)\right]=\frac{\int_{W} v(t, x) \mathrm{d} \mu_{0}}{\int_{W} \bar{v}(t, x) \mathrm{d} \mu_{0}}
$$

where $v$ solves (2.2) with $v_{0}=F$ and $\phi=0$, while $\bar{v}$ solves it with $v_{0}=1$ and $\phi=0$. For brevity, let

$$
\hat{F}_{k}=\int F u_{k} \mathrm{~d} \mu, \quad \hat{1}_{k}=\int u_{k} \mathrm{~d} \mu, \quad \hat{\mu}_{0, k}=\int u_{k} \mathrm{~d} \mu_{0} .
$$

Expressing $v$ and $\bar{v}$ as series solutions using (2.6), we have

$$
v(t, x)=\sum_{k=1}^{\infty} \mathrm{e}^{-\lambda_{k} t} \hat{F}_{k} u_{k}(x), \quad \bar{v}(t, x)=\sum_{k=1}^{\infty} \mathrm{e}^{-\lambda_{k} t} \hat{1}_{k} u_{k}(x) .
$$

After a bit of rearrangement, the error can be expressed as

$$
\begin{aligned}
e(t) & \equiv\left|\mathbb{E}^{\mu_{t}}\left[f\left(T, X_{T}\right)\right]-\mathbb{E}^{\nu}\left[f\left(T, X_{T}\right)\right]\right| \\
& =\left|\frac{\sum_{k} \mathrm{e}^{-\left(\lambda_{k}-\lambda_{1}\right) t}\left(\hat{F}_{k}-\hat{1}_{k} \int F \mathrm{~d} \nu\right) \hat{\mu}_{0, k}}{\hat{1}_{1} \hat{\mu}_{0,1}+\sum_{k} \mathrm{e}^{-\left(\lambda_{k}-\lambda_{1}\right) t} \hat{1}_{k} \hat{\mu}_{0, k}}\right|
\end{aligned}
$$


where the sums are from $k=2$ to $\infty$ since $\hat{F}_{1}=\hat{1}_{1} \int_{W} F \mathrm{~d} \nu$. Noting that

$$
\begin{aligned}
\left|\hat{F}_{k}-\hat{1}_{k} \int F \mathrm{~d} \nu\right| & \leq \int\left|F u_{k}\right| \mathrm{d} \mu+\int|F| \mathrm{d} \nu \int\left|u_{k}\right| \mathrm{d} \mu \\
& \leq 2\|f\|_{L^{\infty}} \int\left|u_{k}\right| \mathrm{d} \mu \leq 2\|f\|_{L^{\infty}} \sqrt{\mu(W)}
\end{aligned}
$$

we can rewrite the numerator as

$$
\begin{aligned}
\left|\sum_{k=2}^{\infty} \mathrm{e}^{-\left(\lambda_{k}-\lambda_{1}\right) t}\left(\hat{F}_{k}-\hat{1}_{k} \int F \mathrm{~d} \nu\right) \hat{\mu}_{0, k}\right| & \leq 2 \sqrt{\mu(W)}\|f\|_{L^{\infty}} \sum_{k=2}^{\infty} \mathrm{e}^{-\left(\lambda_{k}-\lambda_{1}\right) t}\left|\hat{\mu}_{0, k}\right| \\
& \leq 2 \sqrt{\mu(W)}\|f\|_{L^{\infty}} \mathrm{e}^{-\left(\lambda_{2}-\lambda_{1}\right)\left(t-t_{1}\right)} \sum_{k=2}^{\infty} \mathrm{e}^{-\left(\lambda_{k}-\lambda_{1}\right) t_{1}}\left|\hat{\mu}_{0, k}\right| \\
& \leq 2 \sqrt{\mu(W)}\|f\|_{L^{\infty}} \mathrm{e}^{-\left(\lambda_{2}-\lambda_{1}\right)\left(t-t_{1}\right)} \sum_{k=2}^{\infty} \mathrm{e}^{-\kappa \lambda_{k} t_{1}}\left|\hat{\mu}_{0, k}\right|
\end{aligned}
$$

where $\kappa=1-\lambda_{1} / \lambda_{2}$ and $t \geq t_{1}>0$. Applying Proposition A.2 from the appendix to this, the numerator is bounded by

$$
\begin{aligned}
& \left|\sum_{k=2}^{\infty} \mathrm{e}^{-\left(\lambda_{k}-\lambda_{1}\right) t}\left(\hat{F}_{k}-\hat{1}_{k} \int F \mathrm{~d} \nu\right) \hat{\mu}_{0, k}\right| \\
& \quad \lesssim\left\|P_{[2, \infty)} \mu_{0}\right\|_{H_{\mu}^{-s}}\|f\|_{L^{\infty}} \mathrm{e}^{-\left(\lambda_{2}-\lambda_{1}\right)\left(t-t_{1}\right)} t_{1}^{-n / 4-s / 2} .
\end{aligned}
$$

The constant that has been absorbed into the $\lesssim$ symbol is independent of $t, f$ and $\mu_{0}$.

To ensure the denominator is uniformly bounded away from zero, we use a similar treatment,

$$
\begin{aligned}
\sum_{k=2}^{\infty} \mathrm{e}^{-\left(\lambda_{k}-\lambda_{1}\right) t} \hat{1}_{k} \hat{\mu}_{0, k} & \leq \sqrt{\mu(W)} \mathrm{e}^{-\left(\lambda_{2}-\lambda_{1}\right)\left(t-t_{2}\right)} \sum_{k=2}^{\infty} \mathrm{e}^{-\kappa \lambda_{k} t_{2}}\left|\hat{\mu}_{0, k}\right| \\
& \lesssim\left\|P_{[2, \infty)} \mu_{0}\right\|_{H_{\mu}^{-s}} \mathrm{e}^{-\left(\lambda_{2}-\lambda_{1}\right) t} t_{2}^{-n / 4-s / 2}
\end{aligned}
$$

for $t \geq t_{2}>0$, which may differ from $t_{1}$. Therefore,

$$
\begin{aligned}
& \hat{1}_{1} \hat{\mu}_{0,1}+\sum_{k=2}^{\infty} \mathrm{e}^{-\left(\lambda_{k}-\lambda_{1}\right) t} \hat{1}_{k} \hat{\mu}_{0, k} \\
& \quad \gtrsim \hat{1}_{1} \hat{\mu}_{0,1}-\mathrm{e}^{-\left(\lambda_{2}-\lambda_{1}\right)\left(t-t_{2}\right)} t_{2}^{-n / 4-s / 2}\left\|P_{[2, \infty)} \mu_{0}\right\|_{H_{\mu}^{-s}} .
\end{aligned}
$$

For a sufficiently large $t \geq \underline{t} \geq t_{2}>0$, the denominator is bounded from below by

$$
\hat{1}_{1} \hat{\mu}_{0,1}+\sum_{k=2}^{\infty} \mathrm{e}^{-\left(\lambda_{k}-\lambda_{1}\right) t} \hat{1}_{k} \hat{\mu}_{0, k} \geq \frac{1}{2} \hat{1}_{1} \hat{\mu}_{0,1}=\frac{1}{2} \int u_{1} \mathrm{~d} \mu \int u_{1} \mathrm{~d} \mu_{0}>0 .
$$

Roughly,

$$
\underline{t} \gtrsim\left\{\left\|P_{[2, \infty)} \mu_{0}\right\|_{H_{\mu}^{-s}} / \int u_{1} \mathrm{~d} \mu_{0}\right\}^{4 /(n+2 s)} .
$$

Taking $t_{1}=t_{2}=\underline{t}$ in (3.8) and (3.9) we have that for $t \geq \underline{t}$

$$
\begin{aligned}
e(t) \lesssim & \left(\int u_{1} \mathrm{~d} \mu_{0}\right)^{-1} \mathrm{e}^{-\left(\lambda_{2}-\lambda_{1}\right)(t-\underline{t})}(\underline{t})^{-n / 4-s / 2} \\
& \times\|f\|_{L^{\infty}}\left\|P_{[2, \infty)} \mu_{0}\right\|_{H_{\mu}^{-s}}
\end{aligned}
$$


Finally, for this estimate to hold for general bounded and measurable $f$, we apply a density argument with respect to the $L^{\infty}$ norm.

The inclusion of $\int u_{1} \mathrm{~d} \mu_{0}$ in the preceding result is deliberate as $\mu_{0}$ is, to a degree, a user specified parameter. Moreover, $\int u_{1} \mathrm{~d} \mu_{0}$ could be quite small. Indeed, when a $X_{t}$ first enters $W$, it is near $\partial W$ and the support of $\mu_{0}$ is in a neighborhood of $\partial W$; we may have $\mu_{0}=\delta_{x}$ where $x$ is close to $\partial W$. As $u_{1}$ is continuous and vanishes on $\partial W$,

$$
\int_{W} u_{1} \delta_{x}=\mathrm{O}(\operatorname{dist}(x, \partial W))
$$

We also see that as $\mu_{0} \rightarrow \nu,\left\|P_{[2, \infty)} \mu_{0}\right\|_{H_{\mu}^{-s}} \rightarrow 0$, and the error vanishes.

It remains to identify distributions and values of $s$ for which $\left\|\mu_{0}\right\|_{H_{\mu}^{-s}}<\infty$. In the case that $\mu_{0}$ has an $L_{\mu}^{2}$ Radon-Nikodym derivative, one readily sees that $\left\|\mu_{0}\right\|_{H_{\mu}^{-s}}<\infty$ for $s \leq 0$. Indeed, when $s=0$, this results collapses onto the $L_{\mu}^{2}$ estimate of [4]. This extends to $\mu_{0}$ possessing $L_{\mu}^{p}$ densities for any $p \geq 2$.

For the case $\mu_{0}=\delta_{x}$, a Dirac mass, we shall have that $\mu_{0} \in H_{\mu}^{-s}$ when $s$ is large enough to embed $H_{\mu}^{s}$ into $L^{\infty}$. If the $\partial W$ is sufficiently smooth, then by standard elliptic theory, $H_{\mu}^{s}$ and $H^{s}$ will be equivalent for $s \geq 0$, and we have the embedding for $s>n / 2,[1,13,14]$. Refined elliptic estimates may weaken such assumptions on the boundary.

\subsection{Exit times}

In the case that we are interested in exit times, we have a result closely related to Theorem 3.1.

Theorem 3.2. Assume $\mu_{0}$ satisfies the assumptions of Theorem 3.1 and $t_{0} \geq \underline{t}$. Then for $t \geq 0$,

$$
\left|\mathbb{P}^{\mu_{t_{0}}}[T>t]-\mathrm{e}^{-\lambda_{1} t}\right| \leq C \mathrm{e}^{-\lambda_{1} t} \mathrm{e}^{-\left(\lambda_{2}-\lambda_{1}\right) t_{0}}
$$

where $C$ is the pre-exponential factor in (3.2) and is independent of $t$ and $t_{0}$.

Proof. As before, we rely on (2.3) and the series expansions (2.6) to write

$$
\mathbb{P}^{\mu_{t_{1}}}[T>t]=\frac{\mathbb{P}^{\mu_{0}}\left[T>t_{1}+t\right]}{\mathbb{P}^{\mu_{0}}\left[T>t_{1}\right]}=\frac{\sum_{k=1}^{\infty} \mathrm{e}^{-\lambda_{k}\left(t+t_{1}\right)} \hat{1}_{k} \hat{\mu}_{0, k}}{\sum_{k=1}^{\infty} \mathrm{e}^{-\lambda_{k} t_{1}} \hat{1}_{k} \hat{\mu}_{0, k}} .
$$

Comparing against the QSD,

$$
\begin{aligned}
\left|\mathbb{P}^{\mu_{t_{1}}}[T>t]-\mathrm{e}^{-\lambda_{1} t}\right| & =\left|\frac{\sum_{k=1}^{\infty} \mathrm{e}^{-\lambda_{k}\left(t+t_{1}\right)} \hat{1}_{k} \hat{\mu}_{0, k}}{\sum_{k=1}^{\infty} \mathrm{e}^{-\lambda_{k} t_{1}} \hat{1}_{k} \hat{\mu}_{0, k}}-\mathrm{e}^{-\lambda_{1} t}\right| \\
& =\left|\frac{\sum_{k=1}^{\infty}\left(\mathrm{e}^{-\lambda_{k}\left(t+t_{1}\right)}-\mathrm{e}^{-\lambda_{k} t_{1}-\lambda_{1} t}\right) \hat{1}_{k} \hat{\mu}_{0, k}}{\sum_{k=1}^{\infty} \mathrm{e}^{-\lambda_{k} t_{1}} \hat{1}_{k} \hat{\mu}_{0, k}}\right|
\end{aligned}
$$

In the numerator, the $k=1$ term vanishes, leaving

$$
\mathrm{e}^{-\lambda_{1} t}\left|\frac{\sum_{k=2}^{\infty}\left(1-\mathrm{e}^{-\left(\lambda_{k}-\lambda_{1}\right) t}\right) \mathrm{e}^{-\lambda_{k} t_{1}} \hat{1}_{k} \hat{\mu}_{0, k}}{\sum_{k=1}^{\infty} \mathrm{e}^{-\lambda_{k} t_{1}} \hat{1}_{k} \hat{\mu}_{0, k}}\right| \leq \mathrm{e}^{-\lambda_{1} t} \frac{\sum_{k=2}^{\infty} \mathrm{e}^{-\lambda_{k} t_{0}}\left|\hat{1}_{k} \hat{\mu}_{0, k}\right|}{\left|\sum_{k=1}^{\infty} \mathrm{e}^{-\lambda_{k} t_{0}} \hat{1}_{k} \hat{\mu}_{0, k}\right|}
$$

Using the same methods as in the Proof of Theorem 3.1,

$$
\frac{\sum_{k=2}^{\infty} \mathrm{e}^{-\lambda_{k} t_{1}}\left|\hat{\mu}_{0, k}\right|}{\left|\sum_{k=1}^{\infty} \mathrm{e}^{-\lambda_{k} t_{1}} \hat{1}_{k} \hat{\mu}_{0, k}\right|} \lesssim\left(\int u_{1} \mathrm{~d} \mu_{0}\right)^{-1} \underline{t}^{-n / 4-s / 2} \mathrm{e}^{-\left(\lambda_{2}-\lambda_{1}\right)\left(t_{0}-\underline{t}\right)}\left\|P_{[2, \infty)} \mu_{0}\right\|_{H_{\mu}^{-s}}
$$


This estimate plays an important role in our analysis of ParRep. Indeed, we will frequently confront terms of the form $\mathbb{E}^{\mu_{t_{0}}}\left[f(X, T) 1_{T>t}\right]$, and we will want to compare against the corresponding term for the QSD. One could naively apply Theorem 3.1 to estimate such a term, with observable $g_{t}(\xi, \tau)=f(\xi, \tau) 1_{\tau>t}$. However, this is wasteful, as the observable is going to be taken over realizations which not only have not left the well before $t_{0}$, but remain in the well for at least an additional $t$. We thus have the following identity.

Lemma 3.3. Given $t, t_{0} \geq 0$,

$$
\mathbb{E}^{\mu_{t_{0}}}\left[f\left(X_{T}, T\right) 1_{T>t}\right]=\mathbb{E}^{\mu_{t_{0}+t}}\left[f\left(X_{T}, T+t\right)\right] \mathbb{P}^{\mu_{t_{0}}}[T>t] .
$$

Proof. This reflects the Markovian nature of the process. Writing out the lefthand side,

$$
\begin{aligned}
\mathbb{E}^{\mu_{t_{0}}\left[f\left(X_{T}, T\right) 1_{T>t}\right]} & =\int_{W} \mathbb{E}^{x}\left[f\left(X_{T}, T\right) 1_{T>t}\right] \mu_{t_{0}}(\mathrm{~d} x) \\
& =\frac{\int_{W} \mathbb{E}^{x}\left[f\left(X_{T}, T\right) 1_{T>t}\right] \mathbb{P}^{\mu_{0}}\left[X_{t} \in \mathrm{d} x, T>t_{0}\right]}{\mathbb{P}^{\mu_{0}}\left[T>t_{0}\right]}
\end{aligned}
$$

The numerator is

$$
\begin{aligned}
\int_{W} \mathbb{E}^{x}\left[f\left(X_{T}, T\right) 1_{T>t}\right] \mathbb{P}^{\mu_{0}}\left[X_{t} \in \mathrm{d} x, T>t_{0}\right] & =\mathbb{E}^{\mu_{0}}\left[f\left(X_{T}, T-t_{0}\right) 1_{T-t_{0}>t} 1_{T>t_{0}}\right] \\
& =\mathbb{E}^{\mu_{0}}\left[f\left(X_{T}, T-t_{0}\right) 1_{T>t_{0}+t}\right],
\end{aligned}
$$

where $t_{0}$ is subtracted off to make the observable consistent. The same argument shows

$$
\mathbb{E}^{\mu_{t_{0}+t}}\left[f\left(X_{T}, T+t\right)\right]=\frac{\mathbb{E}^{\mu_{0}}\left[f\left(X_{T}, T-t_{0}\right) 1_{T>t+t_{0}}\right]}{\mathbb{P}^{\mu_{0}}\left[T>t+t_{0}\right]} .
$$

Combining these three expressions completes the proof.

In principle, we can use this lemma and Theorem 3.2 to obtain refinements on Theorem 3.1 for observables that include $1_{T>t}$ terms.

\section{The Dephasing SteP - Proof of Theorem 1.2}

We now examine our dephasing step,

Theorem 4.1. Given $s \geq 0$, assume supp $\mu_{\text {phase }}^{0} \subset W$ and $\left\|\mu_{\text {phase }}^{0}\right\|_{H_{\mu}^{-s}}<\infty$. Then

A. The dephasing step produces $N$ independent replicas with distributions $\mu_{\text {phase, }}$

$$
\mu_{\text {phase }}(A)=\mathbb{P}^{\mu_{\text {phase }}^{0}}\left[X_{t_{\text {phase }}} \in A \mid T>t_{\text {phase }}\right]
$$

B. There exists $\underline{t}_{\text {phase }}$ and $C_{\text {phase }}$ such that for $t_{\text {phase }} \geq \underline{t}_{\text {phase }}$,

$$
\left|\mathbb{E}^{\mu_{\text {phase }}}\left[f\left(T^{k}, X_{T^{k}}^{k}\right)\right]-\mathbb{E}^{\nu}\left[f\left(T, X_{T}\right)\right]\right| \leq\|f\|_{L^{\infty}} C_{\text {phase }} \mathrm{e}^{-\left(\lambda_{2}-\lambda_{1}\right) t_{\text {phase }}} ;
$$

for any bounded measurable $f: \mathbb{R}^{+} \times \partial W \rightarrow \mathbb{R}$ and all $k=1, \ldots, N$.

C. The expected number of times a replica is relaunched is finite.

To prove Theorem 4.1, we must establish:

A. The replicas are independent and have law $\mu_{\text {phase }}$;

B. The error of $\mu_{\text {phase }}$ can be made small;

C. The expected number of relaunches is finite. 
The first property is obvious as each of the replicas is driven by an independent Brownian motion, and we only retain realizations for which $T>t_{\text {phase }}$. The second property follows from Theorem 3.1.

To prove the third property, we must establish that replicas initiated from $\mu_{\text {phase }}^{0}$ have a nonzero chance of surviving till $t_{\text {phase: }}$

Lemma 4.2. Assume that $\mu_{\text {phase }}^{0}$ satisfies the hypotheses of Theorem 4.1,

$$
\mathbb{P}^{\mu_{\text {phase }}^{0}}\left[T^{k} \geq t_{\text {phase }}\right] \equiv p>0 \text {. }
$$

Proof. Observe that we have the following monotonicity property for $t_{2}>t_{1}$,

$$
0 \leq \mathbb{P}^{\mu_{\text {phase }}^{0}}\left[T \geq t_{2}\right] \leq \mathbb{P}^{\mu_{\text {phase }}^{0}}\left[T \geq t_{1}\right]
$$

We now argue by contradiction. Assume that at some $t_{1}>0, \mathbb{P}_{\text {phase }}^{0}\left[T \geq t_{1}\right]=0$. By the above monotonicity, $\mathbb{P}^{\mu_{\text {phase }}^{0}}\left[T \geq t_{2}\right]=0$ for all $t_{2} \geq t_{1}$. Using a similar approach as in the Proof of Theorem 3.1, we write

$$
\mathbb{P}^{\mu_{\text {phase }}^{0}}[T \geq t]=\bar{v}(x, t)=\sum_{k=1}^{\infty} \mathrm{e}^{-\lambda_{k} t} \int u_{k} d \mu_{\text {phase }}^{0} \int u_{k} \mathrm{~d} \mu
$$

where $\bar{v}$ solves (2.2) with $v_{0}=1$ and $\phi=0$. Therefore,

$$
\begin{aligned}
\bar{v}\left(x, t_{2}\right) & =\sum_{k=1}^{\infty} \mathrm{e}^{-\lambda_{k} t_{2}} \hat{\mu}_{\text {phase }, k}^{0} \int u_{k} \mathrm{~d} \mu=\sum_{k=1}^{\infty} \mathrm{e}^{-\lambda_{k} t_{2}} \hat{\mu}_{\text {phase }, k}^{0} \hat{1}_{k} \\
& \geq \mathrm{e}^{-\lambda_{1} t_{2}}\left\{\hat{\mu}_{\text {phase }, 1}^{0} \hat{1}_{1}-\mathrm{e}^{-\left(\lambda_{2}-\lambda_{1}\right) t_{2}} \sum_{k=2}^{\infty} \mathrm{e}^{-\kappa \lambda_{k} t_{2}}\left|\hat{1}_{k}\right|\left|\hat{\mu}_{\text {phase }, k}^{0}\right|\right\} \\
& \gtrsim \mathrm{e}^{-\lambda_{1} t_{2}}\left\{\hat{\mu}_{\text {phase }, 1}^{0} \hat{1}_{1}-\mathrm{e}^{-\left(\lambda_{2}-\lambda_{1}\right) t_{2}} t_{2}^{-n / 4-s / 2}\left\|P_{[2, \infty)} \mu_{\text {phase }}^{0}\right\|_{H_{\mu}^{-s}}\right\}
\end{aligned}
$$

Then taking $t_{2}$ sufficiently large,

$$
\begin{aligned}
& \hat{\mu}_{\text {phase }, 1}^{0} \hat{1}_{1}-\mathrm{e}^{-\left(\lambda_{2}-\lambda_{1}\right) t_{2}} t_{2}^{-n / 4-s / 2}\left\|P_{[2, \infty)} \delta_{x}\right\|_{H_{\mu}^{-s}} \\
& \geq \frac{1}{2} \hat{\mu}_{\text {phase }, 1}^{0} \hat{1}_{1}=\frac{1}{2} \int u_{1} \mathrm{~d} \mu_{\text {phase }}^{0} \int u_{1} \mathrm{~d} \mu>0,
\end{aligned}
$$

since $\int u_{1} \mathrm{~d} \mu_{\text {phase }}^{0}>0$. Thus, we have a contradiction.

This calculation reveals a role played by the choice of $\mu_{\text {phase }}^{0}$. If concentrated near the well boundary, $\mathbb{P}^{\mu_{\text {phase }}^{0}}\left[T^{k} \geq t_{\text {phase }}\right]=p$ could be quite small. This will induce the replicas to relaunch many times, as the next result shows. Thus, for computational efficiency, a distribution concentrated deep in the well's interior is desirable.

Lemma 4.3. Assume that $\mu_{\text {phase }}^{0}$ satisfies the hypotheses of Theorem 4.1, and that $\mathbb{P}^{\mu_{\text {phase }}^{0}}\left[T^{k} \geq t_{\text {phase }}\right]=p>$ 0. Then

$$
\mathbb{E}^{\mu_{\text {phase }}^{0}}[\text { Number of relaunches }]=(1-p) / p<\infty
$$

Proof. The probability of relaunching $m$ times is the probability of exiting $m$ times and surviving on the $m+1-$ th time. Interpreting this in terms of $T^{k}$ and using the assumption, $\mathbb{P}^{\mu_{\text {phase }}^{0}}[m$ relaunches $]=(1-p)^{m} p$. Thus,

$$
\begin{aligned}
\mathbb{E}^{\mu_{\text {phase }}^{0}}[\text { Number of relaunches }] & =\sum_{m=0}^{\infty} m \cdot \mathbb{P}^{\mu_{\text {phase }}^{0}}[m \text { relaunches }] \\
& =\sum_{m=0}^{\infty} m(1-p)^{m} p=\frac{1-p}{p}<\infty .
\end{aligned}
$$




\section{The Parallel Step - Proofs of Theorems 1.3 And 1.4}

First, we restate Theorem 1.3 with additional detail:

Theorem 5.1 (Parallel error). Given $t_{\text {phase }} \geq \underline{t}_{\text {phase }}$, let

$$
\epsilon_{\text {phase }} \equiv C_{\text {phase }} \mathrm{e}^{-\left(\lambda_{2}-\lambda_{1}\right) t_{\text {phase }}},
$$

and assume the dephasing step has produced $N$ i.i.d. replicas drawn from distribution $\mu_{\text {phase. }}$ Then the exit time distribution of the parallel step converges to an exponential,

$$
\left|\mathbb{P}^{\mu_{\text {phase }}}\left[T^{\star}>t\right]-\mathrm{e}^{-N \lambda_{1} t}\right| \leq \epsilon_{\text {phase }} N\left(1+\epsilon_{\text {phase }}\right)^{N-1} \mathrm{e}^{-N \lambda_{1} t} .
$$

If $\phi: \partial W \rightarrow \mathbb{R}$ is bounded and measurable, the exit distribution converges to one that is independent of exit time,

$$
\begin{aligned}
& \left|\mathbb{E}^{\mu_{\text {phase }}}\left[1_{T^{\star}>t} \phi\left(X_{T^{\star}}^{\star}\right)\right]-\mathrm{e}^{-N \lambda_{1} t} \int_{\partial W} \phi \mathrm{d} \rho\right| \\
& \lesssim N^{2}\left(1+\epsilon_{\text {phase }}\right)^{N-1} \epsilon_{\text {phase }}\|\phi\|_{L^{\infty}} \mathrm{e}^{-N \lambda_{1} t} .
\end{aligned}
$$

If, in addition, $N \epsilon_{\text {phase }}\left(1+\epsilon_{\text {phase }}\right)^{N-1}<1$, then

$$
\begin{aligned}
& \left|\mathbb{E}^{\mu_{\text {phase }}}\left[\phi\left(X_{T^{\star}}^{\star}\right) \mid T^{\star}>t\right]-\int_{\partial W} \phi \mathrm{d} \rho\right| \\
& \lesssim \frac{N^{2}\|\phi\|_{L^{\infty}} \epsilon_{\text {phase }}\left(1+\epsilon_{\text {phase }}\right)^{N-1}}{1-N \epsilon_{\text {phase }}\left(1+\epsilon_{\text {phase }}\right)^{N-1}} .
\end{aligned}
$$

Proof. To prove (5.1), we begin by writing,

$$
\begin{aligned}
& \left|\mathbb{P}^{\mu_{\text {phase }}}\left[T^{\star}>t\right]-\mathrm{e}^{-N \lambda_{1} t}\right|=\left|\Pi_{k=1}^{N} \mathbb{P}^{\mu_{\text {phase }}}\left[T^{k}>t\right]-\Pi_{k=1}^{N} \mathbb{P}^{\nu}\left[T^{k}>t\right]\right| \\
& =\left|\mathbb{P}^{\mu_{\text {phase }}}\left[T^{1}>t\right]^{N}-\mathrm{e}^{-N \lambda_{1} t}\right| \\
& =\left|\mathbb{P}^{\mu_{\text {phase }}}\left[T^{1}>t\right]-\mathrm{e}^{-\lambda_{1} t}\right|\left|\sum_{k=0}^{N-1} \mathbb{P}^{\mu_{\text {phase }}}\left[T^{1}>t\right]^{k} \mathrm{e}^{-(N-1-k) \lambda_{1} t}\right| \text {. }
\end{aligned}
$$

From Theorem 3.2, we know

$$
\left|\mathbb{P}^{\mu_{\text {phase }}}[T>t]-\mathrm{e}^{-\lambda_{1} t}\right| \leq \epsilon_{\text {phase }} \mathrm{e}^{-\lambda_{1} t}
$$

Therefore,

$$
\left|\mathbb{P}^{\mu_{\text {phase }}}\left[T^{\star} \geq t\right]-\mathrm{e}^{-N \lambda_{1} t}\right| \leq \epsilon_{\text {phase }} \mathrm{e}^{-\lambda_{1} t} N\left(1+\epsilon_{\text {phase }}\right)^{N-1} \mathrm{e}^{-(N-1) \lambda_{1} t} .
$$

To prove (5.2), we begin by writing the expectation as

$$
\begin{aligned}
\mathbb{E}^{\mu_{\text {phase }}\left[1_{T^{\star}>t} \phi\left(X_{T^{\star}}^{\star}\right)\right]} & =\mathbb{E}^{\mu_{\text {phase }}}\left[1_{T^{k_{\star}>t}} \phi\left(X_{T^{k_{\star}}}^{k_{\star}}\right)\right] \\
& =\sum_{k=1}^{N} \mathbb{E}^{\mu_{\text {phase }}}\left[1_{T^{k}>t} \phi\left(X_{T^{k}}^{k}\right) 1_{k=k_{\star}}\right] \\
& =\sum_{k=1}^{N} \mathbb{E}^{\mu_{\text {phase }}}\left[1_{T^{k}>t} \phi\left(X_{T^{k}}^{k}\right) \Pi_{l \neq k} 1_{T^{l}>T^{k}} 1_{T^{l}>t}\right] .
\end{aligned}
$$


In the above expression, we have used that since $T^{\star}>t, T^{l}>t$ for each $l$. Then, using Lemma 3.3 on each of the processes,

$$
\begin{aligned}
& \mathbb{E}^{\mu_{\text {phase }}}\left[1_{T^{k}>t} \phi\left(X_{T^{k}}^{k}\right) \Pi_{l \neq k} 1_{T^{l}>T^{k}} 1_{T^{l}>t}\right]=\mathbb{E}^{\mu_{\text {phase }}+t}\left[\phi\left(X_{T^{k}}^{k}\right) \Pi_{l \neq k} 1_{T^{l}>T^{k}}\right] \Pi_{l=1}^{N} \mathbb{P}^{\mu_{\text {phase }}}\left[T^{l}>t\right] \\
& =\mathbb{E}^{\mu_{t} \text { phase }+t}\left[\phi\left(X_{T^{k}}^{k}\right) 1_{k=k_{\star}}\right] \mathbb{P}^{\mu_{\text {phase }}}[T>t]^{N} \text {. }
\end{aligned}
$$

This leads to the expression

$$
\mathbb{E}^{\mu_{\text {phase }}}\left[1_{T^{\star}>t} \phi\left(X_{T^{\star}}^{\star}\right)\right]=\mathbb{E}^{\mu_{t_{\text {phase }}+t}}\left[\phi\left(X_{T^{\star}}^{\star}\right)\right] \mathbb{P}^{\mu_{\text {phase }}}\left[T^{\star}>t\right] .
$$

Comparing against the QSD,

$$
\begin{aligned}
& \left|\mathbb{E}^{\mu_{\text {phase }}}\left[1_{T^{\star}>t} \phi\left(X_{T^{\star}}^{\star}\right)\right]-\mathbb{E}^{\nu}\left[1_{T^{\star}>t} \phi\left(X_{T^{\star}}^{\star}\right)\right]\right| \leq\left|\mathbb{E}^{\mu_{t_{\text {phase }}+t}}\left[\phi\left(X_{T^{\star}}^{\star}\right)\right]\right|\left|\mathbb{P}^{\mu_{\text {phase }}}\left[T^{\star}>t\right]-\mathrm{e}^{-N \lambda_{1} t}\right| \\
& +\mathrm{e}^{-N \lambda_{1} t}\left|\mathbb{E}^{\mu_{t_{\text {phase }}+t}}\left[\phi\left(X_{T^{\star}}^{\star}\right)\right]-\mathbb{E}^{\nu}\left[\phi\left(X_{T^{\star}}^{\star}\right)\right]\right| \text {. }
\end{aligned}
$$

The first difference can be treated by (5.1), but the second difference requires more care.

Given an arbitrary admissible distribution $\eta$ for $X_{0}$, we define

$$
\mathcal{P}^{\eta}(t) \equiv \mathbb{P}^{\eta}[T>t]=\mathbb{P}^{\eta}\left[T^{k}>t\right], \quad k=1 \ldots N .
$$

Consequently, $\mathcal{P}^{\nu}(t)=\mathrm{e}^{-\lambda_{1} t}$ and

$$
\begin{aligned}
\mathbb{E}^{\mu_{t_{\text {phase }}+t}}\left[\phi\left(X_{T^{\star}}^{\star}\right)\right] & =\sum_{k=1}^{N} \mathbb{E}^{\mu_{t_{\text {phase }}+t}}\left[\phi\left(X_{T}^{k}\right) \Pi_{l \neq k} 1_{T_{l}>T_{k}}\right] \\
& =\sum_{k=1}^{N} \mathbb{E}^{\mu_{t_{\text {phase }}+t}}\left[\phi\left(X_{T}^{k}\right) \mathcal{P}^{\mu_{t_{\text {phase }}+t}}\left(T^{k}\right)^{N-1}\right] .
\end{aligned}
$$

An analogous expansion can be made with $\nu$ in place of $\mu_{t_{\text {phase }}+t}$. Taking the difference of the two sums, and comparing term by term,

$$
\begin{aligned}
& \left|\mathbb{E}^{\mu_{t_{\text {phase }}+t}}\left[\phi\left(X_{T}^{k}\right) \mathcal{P}^{\mu_{t_{\text {phase }}+t}}\left(T^{k}\right)^{N-1}\right]-\mathbb{E}^{\nu}\left[\phi\left(X_{T}^{k}\right) \mathcal{P}^{\nu}\left(T^{k}\right)^{N-1}\right]\right| \\
& \leq\left|\mathbb{E}^{\mu_{t_{\text {phase }}+t}}\left[\phi\left(X_{T}^{k}\right) \mathcal{P}^{\mu_{t_{\text {phase }}+t}}\left(T^{k}\right)^{N-1}\right]-\mathbb{E}^{\nu}\left[\phi\left(X_{T}^{k}\right) \mathcal{P}^{\mu_{t} \text { phase }+t}\left(T^{k}\right)^{N-1}\right]\right| \\
& \quad+\left|\mathbb{E}^{\nu}\left[\phi\left(X_{T}^{k}\right) \mathcal{P}^{\mu_{t_{\text {phase }}+t}}\left(T^{k}\right)^{N-1}\right]-\mathbb{E}^{\nu}\left[\phi\left(X_{T}^{k}\right) \mathcal{P}^{\nu}\left(T^{k}\right)^{N-1}\right]\right| .
\end{aligned}
$$

By Theorem 3.1 the first difference in (5.8) is bounded by

$$
\begin{aligned}
& \left|\mathbb{E}^{\mu_{t_{\text {phase }}+t}}\left[\phi\left(X_{T}^{k}\right) \mathcal{P}^{\mu_{t_{\text {phase }}+t}}\left(T^{k}\right)^{N-1}\right]-\mathbb{E}^{\nu}\left[\phi\left(X_{T}^{k}\right) \mathcal{P}^{\mu_{t_{\text {phase }}+t}}\left(T^{k}\right)^{N-1}\right]\right| \\
& \quad \leq \epsilon_{\text {phase }} \mathrm{e}^{-\left(\lambda_{2}-\lambda_{1}\right) t}\|\phi\|_{L^{\infty}},
\end{aligned}
$$

since $\mathcal{P} \leq 1$.

For the other difference in (5.8), we can replicate the proof of (5.1) to obtain, for any $\tau \geq 0$,

$$
\begin{aligned}
& \left|\mathcal{P}^{\mu_{\text {phase }}+t}(\tau)^{N-1}-\mathcal{P}^{\nu}(\tau)^{N-1}\right| \\
& \leq(N-1) \epsilon_{\text {phase }} \mathrm{e}^{-\left(\lambda_{2}-\lambda_{1}\right) t}\left(1+\epsilon_{\text {phase }} \mathrm{e}^{-\left(\lambda_{2}-\lambda_{1}\right) t}\right)^{N-2} \mathrm{e}^{-(N-1) \lambda_{1} \tau} \\
& \leq(N-1) \epsilon_{\text {phase }}\left(1+\epsilon_{\text {phase }}\right)^{N-2} .
\end{aligned}
$$

Therefore,

$$
\begin{aligned}
& \left|\mathbb{E}^{\nu}\left[\phi\left(X_{T}^{k}\right) \mathcal{P}^{\mu_{t_{\text {phase }}+t}}\left(T^{k}\right)^{N-1}\right]-\mathbb{E}^{\nu}\left[\phi\left(X_{T}^{k}\right) \mathcal{P}^{\nu}\left(T^{k}\right)^{N-1}\right]\right| \\
& \leq\|\phi\|_{L^{\infty}} \epsilon_{\text {phase }}(N-1)\left(1+\epsilon_{\text {phase }}\right)^{N-2}
\end{aligned}
$$


So (5.8) can be bounded by

$$
\begin{aligned}
& \left|\mathbb{E}^{\mu_{t_{\text {phase }}+t}}\left[\phi\left(X_{T}^{k}\right) \mathcal{P}^{\mu_{t_{\text {phase }}+t}}\left(T^{k}\right)^{N-1}\right]-\mathbb{E}^{\nu}\left[\phi\left(X_{T}^{k}\right) \mathcal{P}^{\nu}\left(T^{k}\right)^{N-1}\right]\right| \\
& \leq\|\phi\|_{L^{\infty}} \epsilon_{\text {phase }}\left[1+(N-1)\left(1+\epsilon_{\text {phase }}\right)^{N-2}\right] .
\end{aligned}
$$

Returning to (5.5), using (5.1) to treat the first difference and the preceding calculation to treat the second, we have:

$$
\begin{aligned}
\mid \mathbb{E}^{\mu_{\text {phase }}\left[1_{T^{\star}>t} \phi\left(X_{T^{\star}}^{\star}\right)\right]-\mathbb{E}^{\nu}\left[1_{T^{\star}>t} \phi\left(X_{T^{\star}}^{\star}\right)\right] \mid \leq} & N\|\phi\|_{L^{\infty}} \epsilon_{\text {phase }} \mathrm{e}^{-N \lambda_{1} t}\left(1+\epsilon_{\text {phase }}\right)^{N-1} \\
& +N\|\phi\|_{L^{\infty}} \epsilon_{\text {phase }} \mathrm{e}^{-N \lambda_{1} t}\left[1+(N-1)\left(1+\epsilon_{\text {phase }}\right)^{N-2}\right] \\
\lesssim & N^{2}\|\phi\|_{L^{\infty}} \epsilon_{\text {phase }} \mathrm{e}^{-N \lambda_{1} t}\left(1+\epsilon_{\text {phase }}\right)^{N-1} .
\end{aligned}
$$

Finally, to prove (5.3),

$$
\begin{aligned}
& \left|\mathbb{E}^{\mu_{\text {phase }}}\left[\phi\left(X_{T^{\star}}^{\star}\right) \mid T^{\star}>t\right]-\mathbb{E}^{\nu}\left[\phi\left(X_{T^{\star}}^{\star}\right) \mid T^{\star}>t\right]\right| \\
& =\left|\frac{\mathbb{E}^{\mu_{\text {phase }}}\left[\phi\left(X_{T^{\star}}^{\star}\right) 1_{T^{\star}>t}\right]}{\left.\mathbb{P}_{\text {phase }}^{\mu^{\star}}>t\right]}-\frac{\mathbb{E}^{\nu}\left[\phi\left(X_{T^{\star}}^{\star}\right) 1_{T^{\star}>t}\right]}{\mathbb{P}^{\nu}\left[T^{\star}>t\right]}\right| \\
& \leq\left|\mathbb{E}^{\mu_{\text {phase }}}\left[\phi\left(X_{T^{\star}}^{\star}\right) 1_{T^{\star}>t}\right]-\mathbb{E}^{\nu}\left[\phi\left(X_{T^{\star}}^{\star}\right) 1_{T^{\star}>t}\right]\right| \frac{1}{\mathbb{P}_{\text {phase }}\left[T^{\star}>t\right]} \\
& \quad+\left|\mathbb{E}^{\nu}\left[\phi\left(X_{T^{\star}}^{\star}\right) 1_{T^{\star}>t}\right]\right| \frac{\left|\mathbb{P}^{\mu_{\text {phase }}}\left[T^{\star}>t\right]-\mathbb{P}^{\nu}\left[T^{\star}>t\right]\right|}{\mathbb{P}^{\mu_{\text {phase }}}\left[T^{\star}>t\right] \mathbb{P}^{\nu}\left[T^{\star}>t\right]} .
\end{aligned}
$$

For the first difference,

$$
\begin{aligned}
& \left|\mathbb{E}^{\mu_{\text {phase }}}\left[\phi\left(X_{T^{\star}}^{\star}\right) 1_{T^{\star}>t}\right]-\mathbb{E}^{\nu}\left[\phi\left(X_{T^{\star}}^{\star}\right) 1_{T^{\star}>t}\right]\right| \frac{1}{\mathbb{P}_{\text {phase }}\left[T^{\star}>t\right]} \\
& \lesssim \frac{N^{2}\|\phi\|_{L^{\infty}} \epsilon_{\text {phase }}\left(1+\epsilon_{\text {phase }}\right)^{N-1}}{1-N \epsilon_{\text {phase }}\left(1+\epsilon_{\text {phase }}\right)^{N-1}} .
\end{aligned}
$$

For the second difference,

$$
\begin{aligned}
& \left|\mathbb{E}^{\nu}\left[\phi\left(X_{T^{\star}}^{\star}\right) 1_{T^{\star}>t}\right]\right| \frac{\left|\mathbb{P}^{\mu_{\text {phase }}}\left[T^{\star}>t\right]-\mathbb{P}^{\nu}\left[T^{\star}>t\right]\right|}{\mathbb{P}^{\mu_{\text {phase }}}\left[T^{\star}>t\right] \mathbb{P}^{\nu}\left[T^{\star}>t\right]} \\
& \leq \frac{N\|\phi\|_{L^{\infty}} \epsilon_{\text {phase }}\left(1+\epsilon_{\text {phase }}\right)^{N-1}}{1-N \epsilon_{\text {phase }}\left(1+\epsilon_{\text {phase }}\right)^{N-1}} .
\end{aligned}
$$

Combining these estimates, we have our result.

Lastly, we prove Theorem 1.4, which we first restate with additional detail:

Theorem 5.2 (ParRep error). Let $X_{t}^{\mathrm{s}}$ denote the unaccelerated (serial) process and $X_{t}^{\mathrm{p}}$ denote the ParRep process, and assume that both $X_{t}^{\mathrm{ref}}$ and $X_{t}^{\mathrm{s}}$ are initially distributed under $\mu_{0}$, an admissible distribution. Also assume that $\mu_{\text {phase }}^{0}$ is admissible.

Given $t_{\text {corr }} \geq \underline{t}_{\text {corr }}$ and $t_{\text {phase }} \geq \underline{t}_{\text {phase }}$, let

$$
\begin{aligned}
\epsilon_{\text {corr }} & =C_{\text {corr }} \mathrm{e}^{-\left(\lambda_{2}-\lambda_{1}\right) t_{\text {corr }},} \\
\epsilon_{\text {phase }} & =C_{\text {phase }} \mathrm{e}^{-\left(\lambda_{2}-\lambda_{1}\right) t_{\text {phase }}} .
\end{aligned}
$$

Letting $T^{\mathrm{s}}$ and $T^{\mathrm{p}}$ denote the physical times, we have

$$
\begin{aligned}
& \left|\mathbb{P}^{\mu_{0}}\left[T^{\mathrm{s}}>t\right]-\mathbb{P}^{\mu_{0}}\left[T^{\mathrm{p}}>t\right]\right| \\
& \quad \leq \epsilon_{\text {corr }} \mathrm{e}^{-\lambda_{1} t}+\epsilon_{\text {phase }} N\left(1+\epsilon_{\text {phase }}\right)^{N-1} \mathrm{e}^{-\lambda_{1}\left(t-t_{\text {corr }}\right)_{+},}
\end{aligned}
$$




$$
\begin{aligned}
& \left|\mathbb{E}^{\mu_{0}}\left[\phi\left(X_{T^{\mathrm{s}}}^{\mathrm{s}}\right) 1_{T^{\mathrm{s}}>t}\right]-\mathbb{E}^{\mu_{0}}\left[\phi\left(X_{T^{\mathrm{p}}}^{\mathrm{p}}\right) 1_{T^{\mathrm{p}}>t}\right]\right| \\
& \quad \lesssim\left[\epsilon_{\text {corr }}+\epsilon_{\text {phase }} N^{2}\left(1+\epsilon_{\text {phase }}\right)^{N-1}\right]\|\phi\|_{L^{\infty}} \mathrm{e}^{-\lambda_{1}\left(t-t_{\text {corr }}\right)_{+} .}
\end{aligned}
$$

If, in addition, $\epsilon_{\mathrm{corr}}<1$, then

$$
\begin{gathered}
\left|\mathbb{E}^{\mu_{0}}\left[\phi\left(X_{T^{\mathrm{s}}}^{\mathrm{s}}\right) \mid T^{\mathrm{s}}>t\right]-\mathbb{E}^{\mu_{0}}\left[\phi\left(X_{T^{\mathrm{p}}}^{\mathrm{p}}\right) \mid T^{\mathrm{p}}>t\right]\right| \\
\lesssim \frac{\epsilon_{\text {corr }}+\epsilon_{\mathrm{phase}} N^{2}\left(1+\epsilon_{\mathrm{phase}}\right)^{N-1}}{1-\epsilon_{\text {corr }}}\|\phi\|_{L^{\infty}},
\end{gathered}
$$

Proof. We begin by decomposing

$$
\begin{aligned}
\mathbb{P}^{\mu_{0}}\left[T^{\mathrm{s}}>t\right]= & \mathbb{P}^{\mu_{0}}\left[T^{\mathrm{s}}>t \mid T^{\mathrm{s}} \leq t_{\text {corr }}\right] \mathbb{P}^{\mu_{0}}\left[T^{\mathrm{s}} \leq t_{\text {corr }}\right] \\
& +\mathbb{P}^{\mu_{0}}\left[T^{\mathrm{s}}>t \mid T^{\mathrm{s}}>t_{\text {corr }}\right] \mathbb{P}^{\mu_{0}}\left[T^{\mathrm{s}}>t_{\text {corr }}\right] .
\end{aligned}
$$

We analogously decompose $\mathbb{P}^{\mu_{0}}\left[T^{\mathrm{p}}>t\right]$. For $t \leq t_{\text {corr }}$, the serial algorithm and the reference process of ParRep have the same law. Hence,

$$
\begin{aligned}
\mathbb{P}^{\mu_{0}}\left[T^{\mathrm{s}} \leq t_{\text {corr }}\right] & =\mathbb{P}^{\mu_{0}}\left[T^{\mathrm{p}} \leq t_{\text {corr }}\right] \\
\mathbb{P}^{\mu_{0}}\left[T^{\mathrm{s}}>t \mid T^{\mathrm{s}} \leq t_{\text {corr }}\right] & =\mathbb{P}^{\mu_{0}}\left[T^{\mathrm{p}}>t \mid T^{\mathrm{p}} \leq t_{\text {corr }}\right] .
\end{aligned}
$$

Consequently, error only manifests itself if the parallel step is engaged,

$$
\begin{aligned}
& \left|\mathbb{P}^{\mu_{0}}\left[T^{\mathrm{s}}>t\right]-\mathbb{P}^{\mu_{0}}\left[T^{\mathrm{p}}>t\right]\right| \\
& =\mathbb{P}^{\mu_{0}}\left[T^{\mathrm{s}}>t_{\text {corr }}\right]\left|\mathbb{P}^{\mu_{0}}\left[T^{\mathrm{s}}>t \mid T^{\mathrm{s}}>t_{\text {corr }}\right]-\mathbb{P}^{\mu_{0}}\left[T^{\mathrm{p}}>t \mid T^{\mathrm{p}}>t_{\text {corr }}\right]\right| .
\end{aligned}
$$

Comparing against the QSD,

$$
\begin{aligned}
& \left|\mathbb{P}^{\mu_{0}}\left[T^{\mathrm{s}}>t\right]-\mathbb{P}^{\mu_{0}}\left[T^{\mathrm{p}}>t\right]\right| \\
& \leq \mathbb{P}^{\mu_{0}}\left[T^{\mathrm{s}}>t_{\text {corr }}\right]\left|\mathbb{P}^{\mu_{0}}\left[T^{\mathrm{s}}>t \mid T^{\mathrm{s}}>t_{\text {corr }}\right]-\mathbb{P}^{\nu}\left[T>\left(t-t_{\text {corr }}\right)_{+}\right]\right| \\
& \quad+\mathbb{P}^{\mu_{0}}\left[T^{\mathrm{s}}>t_{\text {corr }}\right]\left|\mathbb{P}^{\mu_{0}}\left[T^{\mathrm{p}}>t \mid T^{\mathrm{p}}>t_{\text {corr }}\right]-\mathbb{P}^{\nu}\left[T>\left(t-t_{\text {corr }}\right)_{+}\right]\right| .
\end{aligned}
$$

Examining the first term,

$$
\mathbb{P}^{\mu_{0}}\left[T^{\mathrm{s}}>t \mid T^{\mathrm{s}}>t_{\mathrm{corr}}\right]=\mathbb{P}^{\mu_{\mathrm{corr}}}\left[T^{\mathrm{s}}>\left(t-t_{\mathrm{corr}}\right)_{+}\right] .
$$

By assumption and Theorem 3.2

$$
\left|\mathbb{P}^{\mu_{\text {corr }}}\left[T^{\mathrm{S}}>\left(t-t_{\text {corr }}\right)_{+}\right]-\mathbb{P}^{\nu}\left[T>\left(t-t_{\text {corr }}\right)_{+}\right]\right| \leq \epsilon_{\text {corr }} \mathrm{e}^{-\lambda_{1}\left(t-t_{\text {corr }}\right)_{+}} .
$$

For the other term, since the exit time is beyond $t_{\text {corr }}$ the parallel step engages. The single reference process is replaced by the ensemble of $N$ replicas drawn from $\mu_{\text {phase }}$, and $T^{\mathrm{p}}=N T^{\star}+t_{\text {corr }}$. Hence,

$$
\mathbb{P}^{\mu_{0}}\left[T^{\mathrm{p}}>t \mid T^{\mathrm{p}}>t_{\text {corr }}\right]=\mathbb{P}^{\mu_{\text {phase }}}\left[T^{\star}>\frac{1}{N}\left(t-t_{\text {corr }}\right)_{+}\right] .
$$

Therefore, by Theorem 5.1

$$
\begin{aligned}
& \left|\mathbb{P}^{\mu_{\text {phase }}}\left[T^{\star}>\frac{1}{N}\left(t-t_{\text {corr }}\right)_{+}\right]-\mathbb{P}^{\nu}\left[T>\left(t-t_{\text {corr }}\right)_{+}\right]\right| \\
& \quad \leq \epsilon_{\text {phase }} N\left(1+\epsilon_{\text {phase }}\right)^{N-1} \mathrm{e}^{-\lambda_{1}\left(t-t_{\text {corr }}\right)_{+}}
\end{aligned}
$$

Substituting (5.14) and (5.15) into (5.13), we obtain (5.10). 
To obtain (5.11), we again decompose as

$$
\begin{aligned}
\mathbb{E}^{\mu_{0}}\left[\phi\left(X_{T^{\mathrm{s}}}^{\mathrm{s}}\right) 1_{T^{\mathrm{s}}>t}\right]= & \mathbb{E}^{\mu_{0}}\left[\phi\left(X_{T^{\mathrm{s}}}^{\mathrm{s}}\right) 1_{T^{\mathrm{s}}>t} \mid T^{\mathrm{s}} \leq t_{\text {corr }}\right] \mathbb{P}^{\mu_{0}}\left[T^{\mathrm{s}} \leq t_{\text {corr }}\right] \\
& +\mathbb{E}^{\mu_{0}}\left[\phi\left(X_{T^{\mathrm{s}}}^{\mathrm{s}}\right) 1_{T^{\mathrm{s}}>t} \mid T^{\mathrm{s}}>t_{\text {corr }}\right] \mathbb{P}^{\mu_{0}}\left[T^{\mathrm{s}}>t_{\text {corr }}\right] .
\end{aligned}
$$

and analogously decompose the ParRep expectation. Again, for $t \leq t_{\text {corr }}$, the serial algorithm and the reference process of ParRep have the same law. Thus

$$
\mathbb{E}^{\mu_{0}}\left[\phi\left(X_{T^{\mathrm{s}}}^{\mathrm{s}}\right) 1_{T^{\mathrm{s}}>t} \mid T^{\mathrm{s}} \leq t_{\text {corr }}\right]=\mathbb{E}^{\mu_{0}}\left[\phi\left(X_{T^{\mathrm{p}}}^{\mathrm{p}}\right) 1_{T^{\mathrm{p}}>t} \mid T^{\mathrm{p}} \leq t_{\text {corr }}\right] .
$$

Consequently,

$$
\begin{aligned}
& \left|\mathbb{E}^{\mu_{0}}\left[\phi\left(X_{T^{\mathrm{s}}}^{\mathrm{s}}\right) 1_{T^{\mathrm{s}}>t}\right]-\mathbb{E}^{\mu_{0}}\left[\phi\left(X_{T^{\mathrm{p}}}^{\mathrm{p}}\right) 1_{T^{\mathrm{p}}>t}\right]\right| \\
& =\mathbb{P}^{\mu_{0}}\left[T^{\mathrm{s}}>t_{\text {corr }}\right]\left|\mathbb{E}^{\mu_{0}}\left[\phi\left(X_{T^{\mathrm{s}}}^{\mathrm{s}}\right) 1_{T^{\mathrm{s}}>t} \mid T^{\mathrm{s}}>t_{\text {corr }}\right]-\mathbb{E}^{\mu_{0}}\left[\phi\left(X_{T^{\mathrm{p}}}^{\mathrm{p}}\right) 1_{T^{\mathrm{p}}>t} \mid T^{\mathrm{p}}>t_{\text {corr }}\right]\right| .
\end{aligned}
$$

Using the QSD as an intermediary,

$$
\begin{aligned}
& \left|\mathbb{E}^{\mu_{0}}\left[\phi\left(X_{T^{\mathrm{s}}}^{\mathrm{s}}\right) 1_{T^{\mathrm{s}}>t} \mid T^{\mathrm{s}}>t_{\text {corr }}\right]-\mathbb{E}^{\mu_{0}}\left[\phi\left(X_{T^{\mathrm{p}}}^{\mathrm{p}}\right) 1_{T^{\mathrm{p}}>t} \mid T^{\mathrm{p}}>t_{\text {corr }}\right]\right| \\
& \leq\left|\mathbb{E}^{\mu_{0}}\left[\phi\left(X_{T^{\mathrm{s}}}^{\mathrm{s}}\right) 1_{T^{\mathrm{s}}>t} \mid T^{\mathrm{s}}>t_{\text {corr }}\right]-\mathbb{E}^{\nu}\left[\phi\left(X_{T}\right) 1_{T>\left(t-t_{\text {corr }}\right)_{+}}\right]\right| \\
& \quad+\left|\mathbb{E}^{\nu}\left[\phi\left(X_{T}\right) 1_{T>\left(t-t_{\text {corr }}\right)_{+}}\right]-\mathbb{E}^{\mu_{0}}\left[\phi\left(X_{T^{\mathrm{p}}}^{\mathrm{p}}\right) 1_{T^{\mathrm{p}}>t} \mid T^{\mathrm{p}}>t_{\text {corr }}\right]\right| .
\end{aligned}
$$

For the first term,

$$
\begin{aligned}
& \mathbb{E}^{\mu_{0}}\left[\phi\left(X_{T^{\mathrm{s}}}^{\mathrm{s}}\right) 1_{T^{\mathrm{s}}>t} \mid T^{\mathrm{s}}>t_{\text {corr }}\right]=\mathbb{E}^{\mu_{\text {corr }}}\left[\phi\left(X_{T^{\mathrm{s}}}^{\mathrm{s}}\right) 1_{T^{\mathrm{s}}>\left(t-t_{\text {corr }}\right)_{+}}\right] \\
& =\mathbb{E}^{\mu_{t_{\text {corr }}+\left(t-t_{\text {corr }}\right)_{+}}\left[\phi\left(X_{T^{\mathrm{s}}}^{\mathrm{s}}\right)\right]} \mathbb{P}^{\mu_{\text {corr }}}\left[T^{\mathrm{s}}>\left(t-t_{\text {corr }}\right)_{+}\right] .
\end{aligned}
$$

Hence,

$$
\begin{aligned}
& \left|\mathbb{E}^{\mu_{0}}\left[\phi\left(X_{T^{\mathrm{s}}}^{\mathrm{s}}\right) 1_{T^{\mathrm{s}}>t} \mid T^{\mathrm{s}}>t_{\text {corr }}\right]-\mathbb{E}^{\nu}\left[\phi\left(X_{T}\right) 1_{T>\left(t-t_{\text {corr }}\right)_{+}}\right]\right| \\
& \lesssim \epsilon_{\text {corr }}\|\phi\|_{L^{\infty}} \mathrm{e}^{-\lambda_{1}\left(t-t_{\text {corr }}\right)_{+}} .
\end{aligned}
$$

For the other term, since the parallel step has engaged,

$$
\mathbb{E}^{\mu_{0}}\left[\phi\left(X_{T^{\mathrm{p}}}^{\mathrm{p}}\right) 1_{T^{\mathrm{p}}>t} \mid T^{\mathrm{p}}>t_{\text {corr }}\right]=\mathbb{E}^{\mu_{\text {phase }}}\left[\phi\left(X_{T^{\star}}^{\star}\right) 1_{T^{\star}>\frac{1}{N}\left(t-t_{\text {corr }}\right)_{+}}\right] .
$$

By Theorem 5.1,

$$
\begin{aligned}
& \left|\mathbb{E}^{\nu}\left[\phi\left(X_{T}\right) 1_{T>\left(t-t_{\text {corr }}\right)_{+}}\right]-\mathbb{E}^{\mu_{0}}\left[\phi\left(X_{T^{\mathrm{p}}}^{\mathrm{p}}\right) 1_{T^{\mathrm{p}}>t} \mid T^{\mathrm{p}}>t_{\text {corr }}\right]\right| \\
& =\left|\mathbb{E}^{\nu}\left[\phi\left(X_{T}\right) 1_{T>\left(t-t_{\text {corr }}\right)_{+}}\right]-\mathbb{E}^{\mu_{\text {phase }}}\left[\phi\left(X_{T^{\star}}^{\star}\right) 1_{T^{\star}>\frac{1}{N}\left(t-t_{\text {corr }}\right)_{+}}\right]\right| \\
& \leq \epsilon_{\text {phase }} N^{2}\|\phi\|_{L^{\infty}}\left(1+\epsilon_{\text {phase }}\right)^{N-1} \mathrm{e}^{-\lambda_{1}\left(t-t_{\text {corr }}\right)_{+}} .
\end{aligned}
$$

Using (5.17) and (5.19) in (5.16) gives (5.11).

(5.12) is proved using the preceding estimates,

$$
\begin{aligned}
\left|\mathbb{E}^{\mu_{0}}\left[\phi\left(X_{T^{\mathrm{s}}}^{\mathrm{s}}\right) \mid T^{\mathrm{s}}>t\right]-\mathbb{E}^{\mu_{0}}\left[\phi\left(X_{T^{\mathrm{p}}}^{\mathrm{p}}\right) \mid T^{\mathrm{p}}>t\right]\right| \\
\leq\left|\frac{\mathbb{E}^{\mu_{0}}\left[\phi\left(X_{T^{\mathrm{s}}}^{\mathrm{s}}\right) 1_{T^{\mathrm{s}}>t}\right]-\mathbb{E}^{\mu_{0}}\left[\phi\left(X_{T^{\mathrm{p}}}^{\mathrm{p}}\right) 1_{T^{\mathrm{p}}>t}\right]}{\mathbb{P}^{\mu_{0}}\left[T^{\mathrm{s}}>t\right]}\right| \\
\quad+\left|\mathbb{E}^{\mu_{0}}\left[\phi\left(X_{T^{\mathrm{p}}}^{\mathrm{p}}\right) 1_{T^{\mathrm{p}}>t}\right]\right| \frac{\mathbb{P}^{\mu_{0}}\left[T^{\mathrm{s}}>t\right]-\mathbb{P}^{\mu_{0}}\left[T^{\mathrm{p}}>t\right]}{\mathbb{P}^{\mu_{0}}\left[T^{\mathrm{s}}>t\right] \mathbb{P}^{\mu_{0}}\left[T^{\mathrm{p}}>t\right]} \mid \\
\lesssim\left[\epsilon_{\text {corr }}+\epsilon_{\text {phase }} N^{2}\left(1+\epsilon_{\text {phase }}\right)^{N-1}\right]\|\phi\|_{L^{\infty}} \frac{\mathrm{e}^{-\lambda_{1}\left(t-t_{\text {corr }}\right)+\mathbb{P}^{\mu_{0}}\left[T^{\mathrm{s}}>t_{\text {corr }}\right]}}{\mathbb{P}^{\mu_{0}}\left[T^{\mathrm{s}}>t\right]} \\
\quad+\left[\epsilon_{\text {corr }}+\epsilon_{\text {phase }} N\left(1+\epsilon_{\text {phase }}\right)^{N-1}\right]\|\phi\|_{L^{\infty}} \frac{\mathrm{e}^{-\lambda_{1}\left(t-t_{\text {corr }}\right)+\mathbb{P}^{\mu_{0}}\left[T^{\mathrm{s}}>t_{\text {corr }}\right]}}{\mathbb{P}_{\mu_{0}}\left[T^{\mathrm{s}}>t\right]} \\
\lesssim\left[\epsilon_{\text {corr }}+\epsilon_{\text {phase }} N^{2}\left(1+\epsilon_{\text {phase }}\right)^{N-1}\right]\|\phi\|_{L^{\infty}} \frac{\mathrm{e}^{-\lambda_{1}\left(t-t_{\text {corr }}\right)+\mathbb{P}^{\mu_{0}}\left[T^{\mathrm{s}}>t_{\text {corr }}\right]}}{\mathbb{P}^{\mu_{0}}\left[T^{\mathrm{s}}>t\right]} .
\end{aligned}
$$


Since $\left(t-t_{\text {corr }}\right)_{+}+t_{\text {corr }} \geq t$

$$
\mathbb{P}^{\mu_{0}}\left[T^{\mathrm{s}}>\left(t-t_{\text {corr }}\right)_{+}+t_{\text {corr }}\right] \leq \mathbb{P}^{\mu_{0}}\left[T^{\mathrm{s}}>t\right] .
$$

Therefore,

$$
\frac{\mathbb{P}^{\mu_{0}}\left[T^{\mathrm{s}}>t_{\mathrm{corr}}\right]}{\mathbb{P}^{\mu_{0}}\left[T^{\mathrm{s}}>t\right]} \leq \frac{\mathbb{P}^{\mu_{0}}\left[T^{\mathrm{s}}>t_{\mathrm{corr}}\right]}{\mathbb{P}^{\mu_{0}}\left[T^{\mathrm{s}}>\left(t-t_{\mathrm{corr}}\right)_{+}+t_{\mathrm{corr}}\right]}=\frac{1}{\mathbb{P}_{\text {corr }}\left[T^{\mathrm{s}}>\left(t-t_{\mathrm{corr}}\right)_{+}\right]}
$$

and

$$
\frac{\mathrm{e}^{-\lambda_{1}\left(t-t_{\mathrm{corr}}\right)+\mathbb{P}^{\mu_{0}}\left[T^{\mathrm{s}}>t_{\mathrm{corr}}\right]}}{\mathbb{P}^{\mu_{0}}\left[T^{\mathrm{s}}>t\right]} \leq \frac{1}{1-\epsilon_{\mathrm{corr}}} .
$$

Substituting this estimate into (5.20) yields (5.12).

\section{Discussion}

We have proven several theorems on the convergence of the exit distributions of parallel replica dynamics to the underlying unaccelerated problem. We have also demonstrated the effectiveness of a dephasing algorithm done in conjunction with the decorrelation step. However, there remain several problems associated with ParRep, both in fully justifying it as an algorithm, and implementing it in practice.

\subsection{Error estimates}

As we pointed out in the introduction, the error estimates in Theorem 1.3 and Theorem 1.4 include terms which grow as $N \rightarrow \infty$. If we take

$$
t_{\text {phase }} \gtrsim k_{\text {phase }} \frac{\log N}{\lambda_{2}-\lambda_{1}}
$$

for some multiplier, $k_{\text {phase }}$, then the most egregious term in the estimates is bounded by

$$
\begin{aligned}
\lim _{N \rightarrow \infty} N^{2} \epsilon_{\text {phase }}\left(1+\epsilon_{\text {phase }}\right)^{N-1} \leq & \lim _{N \rightarrow \infty} C_{\text {phase }} \mathrm{e}^{-k_{\text {phase }} / 2}\left(1+\mathrm{e}^{-k_{\text {phase }}} C_{\text {phase }} / N\right)^{N-1} \\
& =\mathrm{e}^{-k_{\text {phase }} / 2} \mathrm{e}^{C_{\text {phase }} \mathrm{e}^{-k_{\text {phase }}}} .
\end{aligned}
$$

Hence, taking $k_{\text {phase }}$ large enough, the error can be made arbitrarily small. In contrast, the decorrelation error is independent of $N$, and reducing the decorrelation error will not correct for the error due to more replicas.

The error estimate on the exit time in Theorem 1.3 is a bit deceiving and merits additional comment. It would appear that when we consider this cumulative distribution function at any $t>0$, then, sending $N \rightarrow \infty$, the error vanishes. This is a reflection on the estimate being an absolute error. Dividing out by $\mathrm{e}^{-N \lambda_{1} t}$ lets us evaluate the relative error, which we see is uniformly bounded in $t$.

We also remark that since

$$
\mathbb{E}[T]=\int_{0}^{\infty} \mathbb{P}[T>t] \mathrm{d} t,
$$

we can obtain error estimates on the expected exit time. Using the estimates in Theorem 1.3, we see that provided $N \epsilon_{\text {phase }}\left(1+\epsilon_{\text {phase }}\right)^{N-1}<1$, we have

$$
\left|\mathbb{E}^{\mu_{\text {phase }}}\left[T^{\star}\right]-\frac{1}{N \lambda_{1}}\right| \leq N \epsilon_{\text {phase }}\left(1+\epsilon_{\text {phase }}\right)^{N-1} .
$$

Similarly, using the estimates in Theorem 1.4,

$$
\left|\mathbb{E}^{\mu_{0}}\left[T^{\mathrm{s}}\right]-\mathbb{E}^{\mu_{0}}\left[T^{\mathrm{p}}\right]\right| \lesssim \epsilon_{\text {corr }}+N \epsilon_{\text {phase }}\left(1+\epsilon_{\text {phase }}\right)^{N-1} .
$$


It remains to be determined whether our estimates are sharp - is the growth in $N$ real or an artifact of our analysis? While we cannot yet address the sharpness, a simple numerical experiment indicates that there is growth in the error as $N$ increases. Consider the problem

$$
\mathrm{d} X_{t}=-4 X_{t} \mathrm{~d} t+\sqrt{2} \mathrm{~d} B_{t}
$$

for the well $W=[-1,1]$, and suppose we launch $N$ replicas from the Dirac distribution $X_{0}=.1$. By symmetry, we know that if we had perfect dephasing, then during the parallel step

$$
\mathbb{P}^{\nu}\left[X_{T^{\star}}^{\star}=1\right]=\mathbb{P}^{\nu}\left[X_{T^{\star}}^{\star}=-1\right]=\frac{1}{2} .
$$

But if we incompletely dephase, then, because of our asymmetric initial condition, we expect a higher probability of escaping at 1 than -1 . For this problem, we can compute by spectral methods that $\lambda_{1} \approx 0.971972$ and $\lambda_{2} \approx 8.98262$.

To test our conjecture, that the error increases with $N$, we ran 10000 realizations of the dephasing and parallel steps with values of $N=100,200, \ldots, 1000$. We employed Euler-Maruyama time stepping with $\Delta t=10^{-4}$. We then ran this with with $t_{\text {phase }}=.05, .1$ and .2 . The results appear in Figure 3 .

As we predicted, the errors decrease as $t_{\text {phase }}$ increases. For the smallest dephasing time, we also see the error increase with $N$. At $t_{\text {phase }}=.1$, there is still some increase in the error as $N$ increases, though it is less dramatic. When $t_{\text {phase }}=.2$, the trend appears to have been lost to numerical error and sampling variability.

\subsection{Numerical parameters and eigenvalues}

An essential question is how to choose of the dephasing and decorrelation time parameters. Based on the arguments in the preceding section, roughly, if we desire the errors from decorrelation and dephasing to be of the same order, then,

$$
2 \log (N) t_{\text {corr }} \sim t_{\text {phase }} .
$$

So, while they should not be the same, if we can estimate one, we can infer the other. There will also be some mismatch due to different starting distributions for the reference process and the dephasing replicas.

$t_{\text {corr }}$ must be large enough so as to be representative of the QSD while remaining computationally efficient. Taking too large a value of $t_{\text {corr }}$ will just replicate the serial implementation with no acceleration. Theorem 3.1 provides some insight, already discussed in [4]. The error of $\mu_{\text {corr }}$ is controlled by the following quantities:

- The $\mu_{0}$ initial distribution,

- $\left\|P_{[2, \infty)} \mu_{0}\right\|_{H_{\mu}^{-s}}$, the mismatch between the initial distribution of the reference process and the quasistationary distribution, $\nu$;

- The value of $\underline{t}$;

- $\int u_{1} \mathrm{~d} \mu_{0}$

- $\lambda_{2}-\lambda_{1}$, the spectral gap between the first two eigenvalues.

Based on these quantities, and how they relate to $t_{\text {corr }}$, to make the decorrelation error small, we would certainly need

$$
t_{\text {corr }} \gtrsim \frac{\ln \left[\left(\int u_{1} \mathrm{~d} \mu_{0}\right)^{-1}\left\|P_{[2, \infty)} \mu_{0}\right\|_{H_{\mu}^{-s}}\right]}{\lambda_{2}-\lambda_{1}}+\underline{t} .
$$

The eigenvalues also play an important role in determining which problems would benefit from ParRep is an outstanding problem, which is an outstanding issue. For ParRep to be efficient, we need

$$
t_{\text {corr }} \ll \mathbb{E}^{\mu_{\text {corr }}}[T] \sim \mathbb{E}^{\nu}[T]=\frac{1}{\lambda_{1}} .
$$




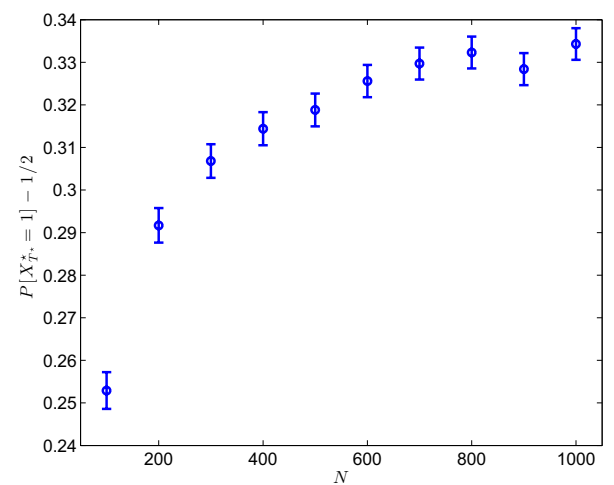

(a) $t_{\text {phase }}=.05$

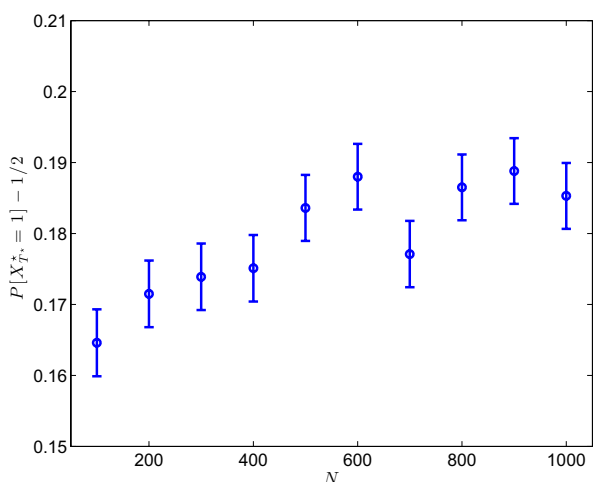

(b) $t_{\text {phase }}=.1$

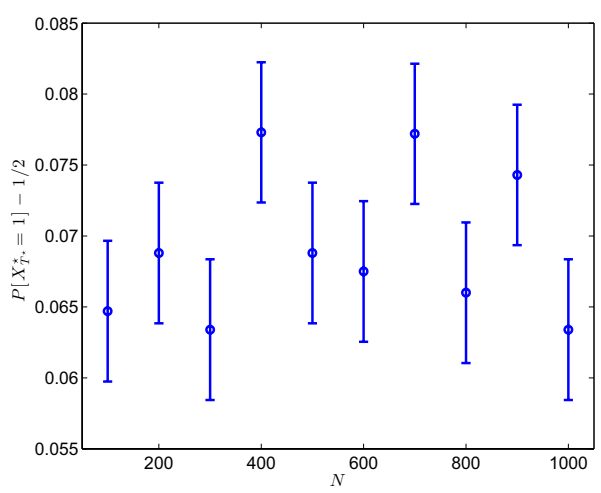

(c) $t_{\text {phase }}=.2$

Figure 3. Three experiments on the impact of imperfect dephasing for (6.3). With perfect dephasing, the probability of exiting at $x=1$ would be $1 / 2$, but because the initial condition is .1 and the dephasing time is finite, there is a persistent bias and growth in the error as $N$ increases. 95\% confidence intervals are plotted for 10000 realizations of each value of $N$.

This is desirable because, in the event $X_{t}$ does not leave the well during the decorrelation step, it is will now take a comparatively long time to exit. In [4], the authors suggested

$$
t_{\text {corr }} \leq \mathbb{E}^{\mu_{0}}[T] .
$$

However, this can be problematic, depending on $\mu_{0}$. As previously discussed, if the replicas launch from a position too close to the boundary, $\mathbb{E}^{\mu_{0}}[T]$ might be rather small. This is mitigated as $t_{\text {corr }}$ becomes larger, leading to $\mathbb{E}^{\mu_{\text {corr }}}[T]$ approaching the escape time of the QSD, $\lambda_{1}^{-1}$.

We can see from (6.4) and constraint (6.5) that ParRep will be most effective when

$$
\frac{1}{\lambda_{2}-\lambda_{1}} \ll \frac{1}{\lambda_{1}},
$$

or, alternatively, when $\lambda_{1} \ll \lambda_{2}-\lambda_{1}$. Under these conditions, $\mu_{\text {corr }}$ converges to $\nu$ much more rapidly than we expect $X_{t}$ to exit $W$. (6.6) can also be viewed as a characterization of when $W$ corresponds to a metastable state for (1.1).

Computing $\lambda_{1}$ and $\lambda_{2}$ directly from a discretization of the elliptic operator $\mathrm{L}$ is intractable for all but the lowest dimensional systems. Instead, one must use Monte Carlo methods, such as those found in $[11,12,17,18,23]$. 
However, these studies, some of which use branching particles processes like Fleming-Viot (discussed below), only yield $\lambda_{1}$.

In a forthcoming work, we explore a mechanism for computing $\lambda_{2}-\lambda_{1}$ using observables. The idea stems from calculations in Theorem 1.1, that, for an observable $\mathcal{O}(x)$, as $t \rightarrow \infty$,

$$
\mathbb{E}^{\mu_{0}}\left[\mathcal{O}\left(X_{t}\right) \mid T>t\right]=\int_{W} \mathcal{O}(x) \mathrm{d} \nu(x)+C\left(\mu_{0}, \mathcal{O}\right) \mathrm{e}^{-\left(\lambda_{2}-\lambda_{1}\right) t}+\ldots
$$

In principle, $\lambda_{2}-\lambda_{1}$ could be extracted from a time series of $\mathbb{E}^{\mu_{0}}\left[\mathcal{O}\left(X_{t}\right) \mid T>t\right]$. This introduces a variety of questions, such as what observables to use and how to perform such a fitting. Thus, we will have a method for dynamically estimating $t_{\text {corr }}$ and $t_{\text {phase }}$.

\subsection{Dephasing mechanism}

The efficiency of our dephasing algorithm can be improved by the availability of multiple processors. For instance, assume we have $N$ processors available for the replicas and that $k$ replicas have successfully been run until $t_{\text {corr }}$. We are still waiting for $N-k$ replicas to successfully dephase. Rather than let $k$ processors sit idle, they could record the successful replicas, and run independent realizations. As more replicas finish dephasing, more processors can be brought to bear on the outstanding replicas.

In practice, as replicas are deemed to have been successfully dephased, they are promoted to the parallel step, [21]. Thus, there is no bottleneck at the dephasing step from waiting to get $N$ realizations dephased.

There are other approaches to dephasing too, such as Fleming-Viot or Moran branching interacting particle processes, $[2,3,9,15]$. These merit consideration for ParRep. These approaches, which randomly split a surviving process every time another process exits the well, can provide additional information, such as an estimate of $\lambda_{1}$. Moreover, no processor sits idle at anytime. However, two challenges are introduced. On a practical level, one needs to implement additional communication routines and synchronization across the processors to request and send configurations as trajectories are killed. The second challenge is analytical, as the dephased processes will now be only approximately independent. This complicates the analysis of the how the error in the dephasing step cascades through the parallel step.

\subsection{Other challenges}

Another task is to assess the cumulative error over many ParRep cycles. The hitting point distribution will be perturbed by the algorithm, meaning that the sequence in which the states are visited would also be perturbed. Quantifying the error across many steps, and showing that it may be made small, would complete the justification of ParRep over the lifetime of a simulation. But to begin such a study, one must decide how to measure

$$
\operatorname{dist}\left(\mathcal{S}_{t}, \mathcal{S}_{t}^{\text {ParRep }}\right) .
$$

The challenge is that $\mathcal{S}_{t}$ is not a Markovian process. A particle that sits near the edge of the well is likely to exit much sooner than one which is near the minima of the well. But that information is lost in the coarse graining. Knowing how long $X_{t}$ has been in the well provides some amount of information; it tells us the proximity to the QSD, from which we can get an exponential exit time.

Despite the challenge of studying the coarse grained flow, we can report that ParRep appears to work as predicted over multiple wells. Consider the flow

$$
\mathrm{d} X_{t}=-2 \pi \sin \left(\pi X_{t}\right) \mathrm{d} t+\sqrt{2} \mathrm{~d} B_{t} .
$$

For this equation, with initial condition $X_{0}=0$, we examined the time it would take to reach the wells centered at $x= \pm 10$. In other words, we sought to compute

$$
T_{ \pm 10}=\inf \left\{t|| X_{t} \mid \geq 9\right\} .
$$




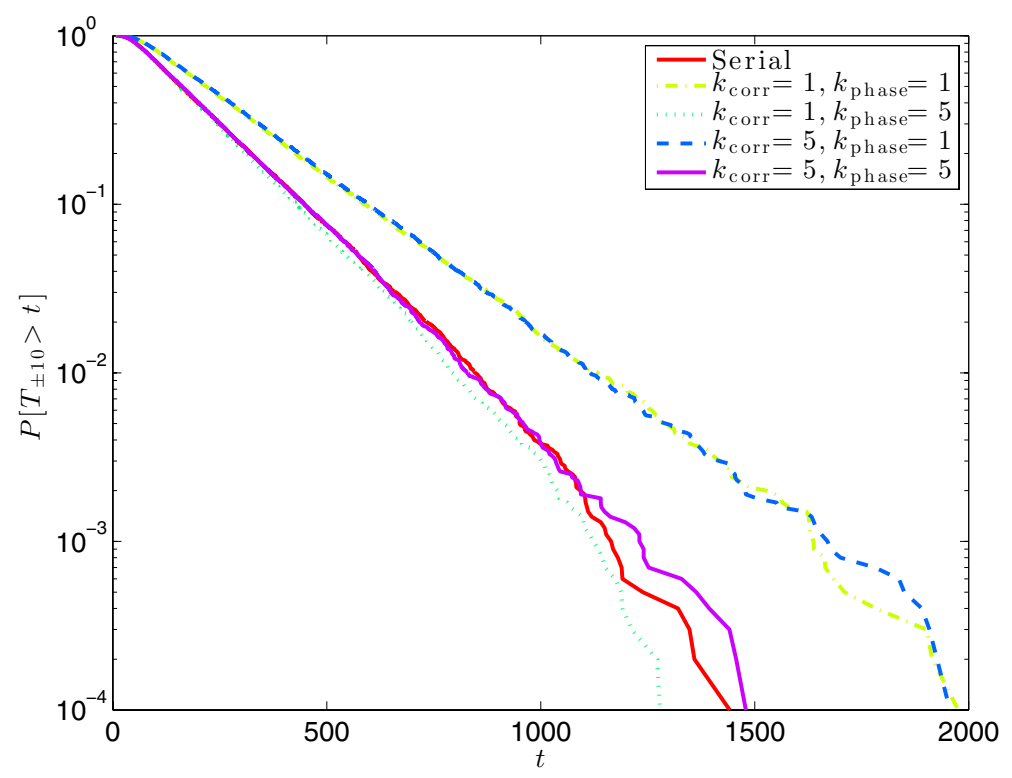

FIgURE 4. The cumulative distribution for the time for it takes trajectory (6.8) to reach the wells centered at \pm 10.10000 realizations of each case were run with time step $\Delta t=10^{-4} \cdot t_{\text {corr }}$ and $t_{\text {phase }}$ relate to $k_{\text {corr }}$ and $k_{\text {phase }}$ via (6.9). As expected, larger values of $t_{\text {corr }}$ and $t_{\text {phase }}$ give better agreement with an unaccelerated process.

For this problem, we ran the full ParRep algorithm (decorrelation, dephasing and parallel steps) within each well. During dephasing, the replicas were initiated from the minima of the present well, $0, \pm 2 \pi, \pm 4 \pi, \ldots$ We ran 10000 realizations of this experiment, varying $k_{\text {corr }}$ and $k_{\text {phase, }}$, where

$$
t_{\text {corr }}=\frac{k_{\text {corr }}}{\lambda_{2}-\lambda_{1}}, \quad t_{\text {phase }}=\frac{k_{\text {phase }}}{\lambda_{2}-\lambda_{1}} .
$$

Since the wells are periodic, we can use spectral methods to compute $\lambda_{1} \approx .202280$ and $\lambda_{2} \approx 16.2588$ once, and we then have these values for all the wells. The results, with $\Delta t=10^{-4}$ and $N=100$ replicas, appear in Figure 4

As we expect, for sufficiently large values of $t_{\text {corr }}$ and $t_{\text {phase }}$, the distributions agree with the serial process. Indeed, in the cases $k_{\text {corr }}=k_{\text {phase }}=5$ and $k_{\text {corr }}=1, k_{\text {phase }}=5$, the exit times agree with the serial realization at $5 \%$ significance level under a Kolmogorov-Smirnov test. In addition, this experiment also supports our calculations that, through the dephasing error, the total error should be magnified by $N$ since increasing the dephasing time improves the fit much more than increasing the decorrelation time does.

Finally, we remark that we have only analyzed the continuous in time problem, though we are ultimately interested in the associated discrete in time algorithm. Much of the analysis carries over to the discrete in time case. A discrete in time quasistationary distribution exists, and there are extensive results on using interacting particle algorithms for dephasing, $[9,10]$. As in the continuous in time case, there remains the subtlety of how to analyze the parallel step when the dephased ensemble is only approximately independent.

However, the discrete time step introduces other subtleties. Assume one uses Euler-Maruyama time discretization with time step $\Delta t$, and define the exit time as

$$
T^{\Delta t}=\inf \left\{t_{n} \mid X_{t_{n}} \notin W\right\} .
$$

For a uniform time step, we see that with no acceleration of the dynamics, the exit times are integer multiples of $\Delta t$. For ParRep, this remains true for exits that take place during the decorrelation step. But for exit times 
taking place during the parallel step, the exit times will be determined by multiples of $N \Delta t$. With a large number of processors, this effective time step could be quite large. When comparing against the continuous in time problem, the error of discretization could be magnified in ParRep. In the preceding experiment, $N \Delta t=.01$, which is small relative to the exit time scale $\left(1 / \lambda_{1} \approx 4.9\right)$ and the decorrelation time scale $\left(1 /\left(\lambda_{2}-\lambda_{1}\right) \approx .062\right)$. Clearly, the discrete in time case warrants a thorough investigation.

\section{Appendix A. Summation bounds}

Much of our analysis relies on bounding series solutions, (2.6), of (2.2), to obtain information about $X_{t}$ through the Feynman-Kac equation, (2.3). The key estimates needed in our work stem from Weyl's Law for $L$ :

Proposition A.1 (Weyl's law for $L$ ). There exist positive constants $c_{1}$ and $c_{2}$, independent of $k$, such that the eigenvalues of (2.1) satisfy

$$
c_{1} k^{2 / n} \leq \lambda_{k} \leq c_{2} k^{2 / n} .
$$

Recall that $n$ denotes the dimension of the underlying problem; $X_{t} \in \mathbb{R}^{n}$.

Proof. We will not reproduce the proof here, which is accomplished by rewriting the eigenvalue problem as

$$
-\beta^{-1} \nabla \cdot\left(\mathrm{e}^{-\beta V} \nabla u\right)=\lambda \mathrm{e}^{-\beta V} u .
$$

This is justified because $V$ is smooth and $W$ is bounded; thus $\mathrm{e}^{-\beta V}$ is smooth and nondegenerate. This is now in the form of Theorem 6.3.1 of [8] on Weyl's Law, yielding the result.

Using Weyl's Law, we have our main summation result,

Proposition A.2. Given $s \geq 0$, let $\mathbf{a}=\left(a_{1}, a_{2}, \ldots\right)$ satisfy

$$
\left\{\sum_{k=1}^{\infty} \lambda_{k}^{-s}\left|a_{k}\right|^{2}\right\}^{1 / 2}=\|\mathbf{a}\|_{H_{\mu}^{-s}}<\infty .
$$

Let $f$ be defined as

$$
f(\tau) \equiv \sum_{k=1}^{\infty} a_{k} \lambda_{k}^{\alpha} \mathrm{e}^{-\tau \lambda_{k}}
$$

For $a>0$, we have:

$A$.

$$
\sup _{\tau \geq a}|f(\tau)| \lesssim a^{-n / 4-\max \{s / 2+\alpha, 0\}}\|\mathbf{a}\|_{H_{\mu}^{-s}}<\infty
$$

B. The convergence of the series is uniform in $\tau \geq a$;

C. $f$ is continuous.

To prove Proposition A.2, we first have the following lemma.

Lemma A.3. Let $\lambda_{k}$ be the eigenvalues and eigenfunctions of $L,(2.1)$. There exists a constant $C>0$, independent of $\tau$, such that for all $\tau>0$,

$$
\sum_{k=1}^{\infty} \lambda_{k}^{\alpha} \mathrm{e}^{-\tau \lambda_{k}} \leq C \tau^{-n / 2-\max \{\alpha, 0\}} .
$$

The reader should rightfully expect the lefthand side of (A.5) to grow as $\alpha \rightarrow \infty$. Indeed, the constant $C$ depends on $\alpha$ and will grow. However, as $\alpha$ is fixed, and we are interested in an estimate in $\tau$, this is suppressed. 
Proof. For $\alpha \leq 0$,

$$
\begin{aligned}
\sum_{k=1}^{\infty} \mathrm{e}^{-\tau \lambda_{k}} \lambda_{k}^{\alpha} & \leq \sum_{k=1}^{\infty} \mathrm{e}^{-\tau \lambda_{k}} \lambda_{1}^{\alpha} \leq \sum_{k=1}^{\infty} \mathrm{e}^{-c_{1} \tau k^{2 / n}} \lambda_{1}^{\alpha} \\
& \leq \lambda_{1}^{\alpha} \int_{0}^{\infty} \mathrm{e}^{-c_{1} \tau k^{2 / n}} \mathrm{~d} k=\lambda_{1}^{\alpha}\left(c_{1} \tau\right)^{-n / 2} \Gamma\left[1+\frac{n}{2}\right] .
\end{aligned}
$$

In the above computation, we approximated the sum as the lower Riemann sum of the integral.

For $\alpha>0$, we begin by estimating

$$
\sum_{k=1}^{\infty} \mathrm{e}^{-\tau \lambda_{k}} \lambda_{k}^{\alpha} \leq \sum_{k=1}^{\infty} \mathrm{e}^{-c_{1} \tau k^{2 / n}} c_{2}^{\alpha} k^{2 \alpha / n} .
$$

For sufficiently large $k$,

$$
k \geq k_{1} \equiv\left\lceil\left(\frac{\alpha}{c_{1} \tau}\right)^{n / 2}\right\rceil,
$$

the summand is monotonically decreasing, while for $k<k_{1}$, it is monotonically increasing. Splitting the sum up,

$$
\begin{aligned}
\sum_{k=1}^{\infty} \mathrm{e}^{-c_{1} \tau k^{2 / n}} k^{2 \alpha / n} & =\sum_{k=1}^{k_{1}} \mathrm{e}^{-c_{1} \tau k^{2 / n}} k^{2 \alpha / n+1}+\sum_{k=k_{1}+1}^{\infty} \mathrm{e}^{-c_{1} \tau k^{2 / n}} k^{2 \alpha / n} \\
& \leq \mathrm{e}^{-c_{1} \tau} \sum_{k=1}^{k_{1}} k^{2 \alpha / n}+\sum_{k=k_{1}+1}^{\infty} \mathrm{e}^{-c_{1} \tau k^{2 / n}} k^{2 \alpha / n} .
\end{aligned}
$$

Crudely bounding the first sum in terms of a max, and treating the latter sum as a lower Riemann approximations of an integral,

$$
\begin{aligned}
& \sum_{k=1}^{\infty} \mathrm{e}^{-c_{1} \tau k^{2 / n}} k^{2 \alpha / n} \leq \mathrm{e}^{-c_{1} \tau} k_{1} \cdot k_{1}^{2 \alpha / n}+\int_{k_{1}}^{\infty} \mathrm{e}^{-c_{1} \tau k^{2 / n}} k^{2 \alpha / n} \mathrm{~d} k \\
& \quad \leq \mathrm{e}^{-c_{1} \tau}\left[\left(\frac{\alpha}{c_{1} \tau}\right)^{n / 2}+1\right]^{2 \alpha / n+1}+\int_{0}^{\infty} \mathrm{e}^{-c_{1} \tau k^{2 / n}} k^{2 \alpha / n} \mathrm{~d} k \\
& \quad \leq\left(\frac{c_{1} \tau}{\alpha}\right)^{-n / 2-\alpha} \mathrm{e}^{-c_{1} \tau}\left[1+\left(\frac{c_{1} \tau}{\alpha}\right)^{n / 2}\right]^{2 \alpha / n+1}+\frac{n}{2}\left(c_{1} \tau\right)^{-n / 2-\alpha} \Gamma\left[\frac{n}{2}+\alpha\right] \\
& \quad \lesssim \tau^{-n / 2-\alpha} .
\end{aligned}
$$

The integrals were computed using Mathematica, with the commands

Integrate $\left[\operatorname{Exp}\left[-c * t * k^{\wedge}(2 / n)\right],\{k, 0, \operatorname{Inf}\right.$ inity $\left.\}\right]$

Integrate $\left[\operatorname{Exp}\left[-\mathrm{c} * \mathrm{t} * \mathrm{k}^{\wedge}(2 / \mathrm{n})\right] * \mathrm{k}^{\wedge}(2 * \mathrm{a} / \mathrm{n}+1),\{\mathrm{k}, 0\right.$, Infinity $\left.\}\right]$

Now we prove Proposition A.2.

Proof. We first observe that $f$ is well defined and bounded:

$$
|f(\tau)| \leq \sum_{k=1}^{\infty}\left|a_{k}\right| \lambda_{k}^{\alpha} \mathrm{e}^{-\tau \lambda_{k}} \leq\left\{\sum_{k=1}^{\infty} \lambda^{s+2 \alpha} \mathrm{e}^{-2 \lambda_{k} \tau}\right\}^{1 / 2}\|\mathbf{a}\|_{H_{\mu}^{-s}} .
$$


Applying Lemma A.3 with $\alpha \mapsto s+2 \alpha$ and $\tau \mapsto 2 a$,

$$
\sum_{k=1}^{\infty} \lambda^{s+2 \alpha} \mathrm{e}^{-2 \lambda_{k} a} \lesssim(2 a)^{-n / 2-\max \{s+2 \alpha, 0\}} .
$$

To prove uniform convergence, let

$$
f_{m}(\tau) \equiv \sum_{k=1}^{m} a_{k} \lambda_{k}^{\alpha} \mathrm{e}^{-\tau \lambda_{k}}
$$

denote the partial sum. Obviously, each partial sum is continuous in $\tau$. Then

$$
\begin{aligned}
\left|f(\tau)-f_{m}(\tau)\right| & \leq \sum_{k=m+1}^{\infty}\left|a_{k}\right| \lambda_{k}^{\alpha} \mathrm{e}^{-\tau \lambda_{k}} \\
& \leq\left\{\sum_{k=m+1}^{\infty} \lambda_{k}^{s+2 \alpha} \mathrm{e}^{-2 \lambda_{k} a}\right\}^{1 / 2}\left\|P_{[m+1, \infty)} \mathbf{a}\right\|_{H_{\mu}^{-s}} \\
& \leq\|\mathbf{a}\|_{H_{\mu}^{-s}}\left\{\sum_{k=m+1}^{\infty} \lambda_{k}^{s+2 \alpha} \mathrm{e}^{-2 \lambda_{k} a}\right\}^{1 / 2} .
\end{aligned}
$$

Examining the sum,

$$
\sum_{k=m+1}^{\infty} \lambda_{k}^{s+2 \alpha} \mathrm{e}^{-2 \lambda_{k} a} \lesssim \sum_{k=m+1}^{\infty} k^{2 s / n+4 \alpha / n} \mathrm{e}^{-2 c_{1} a k^{2 / n}} .
$$

Taking $m$ sufficiently large, the summand will be strictly decreasing in $k$, so we can treat it as a lower Riemann sum for the integral

$$
\int_{m}^{\infty} k^{2 s / n+4 \alpha / n} \mathrm{e}^{-2 c_{1} a k^{2 / n}} \mathrm{~d} k .
$$

Changing variables by letting $k^{2 / n}=l$,

$$
\sum_{k=m+1}^{\infty} \lambda_{k}^{s+2 \alpha} \mathrm{e}^{-2 \lambda_{k} a} \lesssim \int_{m^{2 / n}}^{\infty} l^{s+2 \alpha+n / 2-1} \mathrm{e}^{-2 c_{1} a l} \mathrm{~d} l .
$$

If $s+2 \alpha+n / 2-1 \leq 0$, then

$$
\sum_{k=m+1}^{\infty} \lambda_{k}^{s+2 \alpha} \mathrm{e}^{-2 \lambda_{k} a} \lesssim \int_{m^{2 / n}}^{\infty} \mathrm{e}^{-2 c_{1} a l} \mathrm{~d} l=\frac{1}{2 c_{1} a} \mathrm{e}^{-2 m^{2 / n} c_{1} a} .
$$

On the other hand, if $s+2 \alpha+n / 2-1>0$, we can trade some of the exponential decay to eliminate the algebraic term,

$$
\sum_{k=m+1}^{\infty} \lambda_{k}^{s+2 \alpha} \mathrm{e}^{-2 \lambda_{k} a} \lesssim \int_{m^{2 / n}}^{\infty} \mathrm{e}^{-c_{1} a l} \mathrm{~d} l=\frac{1}{c_{1} a} \mathrm{e}^{-m^{2 / n} c_{1} a} .
$$

In either case, we see that for any $a>0$,

$$
\lim _{m \rightarrow \infty} \sup _{\tau \geq a}\left|f(\tau)-f_{m}(\tau)\right|=0 .
$$

Since the partial sums converge uniformly to $f$, it is now a classical result to conclude that $f$ is continuous for $\tau \geq a>0,[24]$. 
Acknowledgements. The authors wish to thank D. Aristoff, K. Leder, S. Mayboroda, A. Shapeev and O. Zeitouni for helpful conversations in developing these ideas. We also thank D. Perez and A.F. Voter for conversations at LANL that motivated important refinements of our estimates. This work was supported by the NSF PIRE grant OISE-0967140 and the DOE grant DE-SC0002085.

\section{REFERENCES}

[1] R.A. Adams and J.J.F. Fournier, Sobolev spaces. Academic Press 140 (2003).

[2] M. Bieniek, K. Burdzy and S. Finch, Non-extinction of a Fleming-Viot particle model. Probab. Theory Relat. Fields (2011).

[3] M. Bieniek, K. Burdzy and S. Pal, Extinction of Fleming-Viot-type particle systems with strong drift. Electron. J. Prob. 17 (2012).

[4] C. Le Bris, T. Lelièvre, M. Luskin and D. Perez, A mathematical formalization of the parallel replica dynamics. Monte Carlo Methods Appl. 18 (2012) 119-146.

[5] P. Cattiaux, P. Collet, A. Lambert, S. Martínez, S. Méléard and J. San Martín, Quasi-stationary distributions and diffusion models in population dynamics. Ann. Prob. 37 (2009) 1926-1969.

[6] P. Cattiaux and S. Méléard, Competitive or weak cooperative stochastic Lotka-Volterra systems conditioned on non-extinction. J. Math. Biol. 60 (2010) 797-829.

[7] P. Collet, S. Martínez and J. San Martín, Asymptotic laws for one-dimensional diffusions conditioned to nonabsorption. Ann. Prob. 23 (1995) 1300-1314.

[8] E.B. Davies, Spectral theory and differential operators. Cambridge University Press 42 (1996).

[9] P. Del Moral, Feynman-Kac Formulae: Genealogical and Interacting Particle Systems with Applications. Probability and Its Applications, Springer (2011).

[10] P. Del Moral and A. Doucet, Particle motions in absorbing medium with hard and soft obstacles. Stoch. Anal. Appl. 22 (2004) $1175-1207$.

[11] P. Del Moral and L. Miclo, Particle approximations of Lyapunov exponents connected to Schrödinger operators and FeynmanKac semigroups. ESAIM: PS $\mathbf{7}$ (2003) 171-208.

[12] M. El Makrini, B. Jourdain and T. Lelievre, Diffusion Monte Carlo method: Numerical analysis in a simple case. ESAIM: M2AN 41 (2007) 189-213.

[13] L.C. Evans, Partial Differential Equations. Amer. Math. Soc. 2002.

[14] D. Gilbarg and N.S. Trudinger, Elliptic partial differential equations of second order. Springer Verlag 224 (2001).

[15] I. Grigorescu and M. Kang, Hydrodynamic limit for a Fleming-Viot type system. Stoch. Process. Their Appl. 110 (2004) 111-143.

[16] D. Haroske and H. Triebel, Distributions, Sobolev spaces, elliptic equations. Europ. Math. Soc. (2008).

[17] A. Lejay and S. Maire, Computing the principal eigenvalue of the Laplace operator by a stochastic method. Math. Comput. Simul. 73 (2007) 351-363.

[18] A. Lejay and S. Maire, Computing the principal eigenelements of some linear operators using a branching Monte Carlo method. J. Comput. Phys. 227 (2008) 9794-9806.

[19] S. Martínez and J. San Martín, Quasi-stationary distributions for a Brownian motion with drift and associated limit laws. J. Appl. Probab. 31 (1994) 911-920.

[20] S. Martínez and J. San Martín. Classification of killed one-dimensional diffusions. Ann. Probab. 32 (2004) 530-552.

[21] D. Perez, Implementation of Parallel Replica Dynamics, Personal Communication (2012).

[22] D. Perez, B.P. Uberuaga, Y. Shim, J.G. Amar and A.F. Voter, Accelerated molecular dynamics methods: introduction and recent developments. Ann. Reports Comput. Chemistry 5 (2009) 79-98.

[23] M. Rousset, On the control of an interacting particle estimation of Schrödinger ground states. SIAM J. Math. Anal. 38 (2006) 824-844 (electronic).

[24] W. Rudin, Principles of Mathematical Analysis. McGraw-Hill (1976).

[25] D. Steinsaltz and S.N. Evans, Quasistationary distributions for one-dimensional diffusions with killing. Trans. Amer. Math. Soc. 359 (2007) 1285-1324 (electronic).

[26] A.F. Voter, Parallel replica method for dynamics of infrequent events. Phys. Rev. B 57 (1998) 13985-13988.

[27] A.F. Voter, F. Montalenti and T.C. Germann, Extending the time scale in atomistic simulation of materials. Ann. Rev. Materials Sci. 32 (2002) 321-346. 